\title{
Cetuximab-conjugated iron oxide nanoparticles for cancer imaging and therapy
}

\author{
This article was published in the following Dove Press journal: \\ International Journal of Nanomedicine \\ 20 May 2015 \\ Number of times this article has been viewed
}

\author{
Shih-Heng Tseng ${ }^{1,2}$ \\ Min-Yuan Chou ${ }^{2}$ \\ I-Ming Chu'
}

'Department of Chemical Engineering, National Tsing Hua University, ${ }^{2}$ Biomedical Technology and Device Research Laboratories, Industrial Technology Research Institute, Hsinchu, Taiwan
Correspondences: Min-Yuan Chou Biomedical Technology and Device Research Laboratories, Industrial Technology Research Institute, 195, Section 4, Chung Hsing Road, Chutung, Hsinchu 31040, Taiwan

Tel +886359 I 2144

$\mathrm{Fax}+88635910097$

Email minyuanc@itri.org.tw

I-Ming Chu

Department of Chemical Engineering,

National Tsing Hua University,

I0I Section 2, Kuang-Fu Road,

Hsinchu 300 13, Taiwan

Tel +88635713704

Fax +886 357। 5408

Email chuiming123@gmail.com

\begin{abstract}
We have developed a theranostic nanoparticle, ie, cet-PEG-dexSPIONs, by conjugation of the anti-epidermal growth factor receptor (EGFR) monoclonal antibody, cetuximab, to dextran-coated superparamagnetic iron oxide nanoparticles (SPIONs) via periodate oxidation. Approximately 31 antibody molecules were conjugated to each nanoparticle. Cet-PEGdexSPIONs specifically bind to EGFR-expressing tumor cells and enhance image contrast on magnetic resonance imaging. Cet-PEG-dexSPION-treated A431 cells showed significant inhibition of epidermal growth factor-induced EGFR phosphorylation and enhancement of EGFR internalization and degradation. In addition, a significant increase in apoptosis was detected in EGFR-overexpressing cell lines, A431 and 32D/EGFR, after 24 hours of incubation at $37^{\circ} \mathrm{C}$ with cet-PEG-dexSPIONs compared with cetuximab alone. The antibody-dependent cell-mediated cytotoxicity of cetuximab was observed in cet-PEG-dexSPIONs. The results demonstrated that cet-PEG-dexSPIONs retained the therapeutic effect of cetuximab in addition to having the ability to target and image EGFR-expressing tumors. Cet-PEG-dexSPIONs represent a promising targeted magnetic probe for early detection and treatment of EGFR-expressing tumor cells.

Keywords: epidermal growth factor receptor, cetuximab, superparamagnetic iron oxide nanoparticle, magnetic resonance imaging, sodium periodate, polyethylene glycol
\end{abstract}

\section{Introduction}

The human epidermal growth factor receptor (EGFR), a member of the ErbB family of transmembrane receptor tyrosine kinases, consists of an extracellular ligand-binding domain, a hydrophobic transmembrane region, and an intracellular tyrosine kinase domain. Overexpression of EGFR has been documented in approximately one third of epithelial malignancies, including head and neck, colorectal, breast, ovarian, prostate, bladder, and lung cancers. ${ }^{1}$ Aberrant and constitutive EGFR activation contributes to tumor growth and progression, including enhanced cell proliferation, cell cycle progression, tumor angiogenesis, invasion, metastasis, and inhibition of apoptosis. Studies have also reported that high levels of EGFR expression in tumors correlate with a poor prognosis and reduced recurrence-free or overall survival rates..$^{2-4}$

Cetuximab $\left(\mathrm{C} 225\right.$, Erbitux $\left.^{\circledR}\right)$ is a chimeric human/murine monoclonal antibody in the immunoglobulin (Ig)G1 class that specifically targets the human EGFR with a 2-log higher affinity than the natural ligands, transforming growth factor- $\alpha$ and epidermal growth factor. ${ }^{5}$ The US Food and Drug Administration has approved cetuximab as first-line treatment for $K$-ras wild-type EGFR-expressing metastatic colorectal cancer in combination with FOLFIRI (irinotecan, 5-fluorouracil, and leucovorin). ${ }^{6,7}$ Cetuximab is also indicated for the treatment of locally advanced squamous cell carcinoma of the head and neck in combination with radiation therapy and for treatment of recurrent or metastatic squamous cell carcinoma of the head and neck as a single agent. ${ }^{8}$ 
This therapeutic antibody has also shown promising results in preclinical and clinical trials investigating the treatment of different tumor types. ${ }^{9,10}$

Cetuximab blocks ligand binding to and inhibits ligandinduced phosphorylation and activation of the EGFR tyrosine kinase. ${ }^{11}$ The binding of cetuximab to EGFR promotes receptor internalization and subsequent degradation, resulting in downregulation of the receptor. The reduced availability of EGFR on the cell surface effectively prevents activation of EGFR-associated downstream signaling pathways. ${ }^{12,13}$ The antitumor activity of cetuximab has been demonstrated in preclinical models, including G0/G1 cell-cycle arrest, induction of apoptosis, inhibition of DNA repair, inhibition of angiogenesis, and inhibition of tumor cell motility, invasion, and metastasis. ${ }^{14-21}$ Because it has a human IgG1 backbone, cetuximab has also been reported to be capable of having a cytotoxic effect through antibody-dependent cellmediated cytotoxicity (ADCC). ${ }^{22,23}$ In cetuximab-modified nanoparticles, cetuximab can function as a targeting moiety for recognizing EGFR-overexpressing cells, and bring about other therapeutic and diagnostic effects. These effects have been reported in cetuximab-conjugated gold nanoparticles, which are able to target EGFR in vitro and in vivo, leading to an increase in the target/nontarget distribution ratio, enhancing cellular internalization of the targeted nanoparticles, and improving imaging signals. ${ }^{24-26}$

With the advantages of its noninvasive and nondestructive nature and multidimensional tomographic capabilities, coupled with an unparalleled spatial resolution of 10-100 $\mu \mathrm{m}$, magnetic resonance imaging (MRI) has emerged as one of the most powerful imaging modalities in the diagnosis and clinical staging of cancer. MRI has excellent soft tissue contrast and is free of ionizing radiation. However, the low-signal sensitivity of MRI limits its application for early detection of tumors. Thus, the development of targeted MRI contrast agents has become essential for improving the specificity and sensitivity of cancer imaging. Due to their superior magnetic properties, superparamagnetic iron oxide nanoparticles (SPIONs), which are $T_{2}$ contrast agents, can significantly shorten the longitudinal $\left(\mathrm{T}_{1}\right)$ and transverse $\left(\mathrm{T}_{2}\right)$ magnetic relaxation time of water protons, thereby providing a dark negative signal intensity in the images. ${ }^{27}$ SPIONs are completely biodegradable and have excellent biocompatibility. Moreover, compared with the paramagnetic gadolinium chelates, SPIONs have higher sensitivity, lower toxicity, and a longer plasma half-life. In recent years, tremendous effort has been devoted to development of SPION-based nanotheranostics (ie, theranostic nanomedicines) for early detection of cancer cells and targeted therapies based on cancer-specific markers by simultaneously conjugating SPIONs to active targeting moieties, such as ligands and monoclonal antibodies, and to therapeutic agents. ${ }^{28,29}$ By providing real-time feedback of the therapeutic response, such SPION-based nanotheranostics are expected to enable personalized medicine. However, the functionality of the targeting moiety after nanoconjugation needs to be further evaluated.

This study describes the production of cetuximabfunctionalized SPIONs, ie, cet-PEG-dexSPIONs, for targeted MRI and therapy in EGFR-expressing tumor cells. The dextran-coated magnetic nanoprobe surface was modified with a dihydrazide-PEG (polyethylene glycol) linker to reduce nonspecific binding under biological conditions. Further, to retain active antigen-binding sites for efficient targeting, directional conjugation of cetuximab was achieved via the carbohydrate moiety on the heavy chains of the Fc portion of the antibody. The morphology, size, and surface charge of these synthesized magnetic nanoprobes were examined by transmission electron microscopy, dynamic light scattering, and zeta potential measurements. The binding specificity of the contrast agents to differential EGFR-expressing tumor cells was evaluated by immunofluorescence staining, flow cytometry analysis, cellular iron uptake, and in vitro $\mathrm{T}_{2}$-weighted MRI. The therapeutic relevance of the cet-PEG-dexSPIONs for the treatment of EGFR-expressing tumors was evaluated in vitro by Western blot analysis, assessment of downregulation of surface EGFR, analysis of apoptosis, and the ADCC assay.

\section{Materials and methods Cell culture}

The A431 cells were grown in Dulbecco's Modified Eagle's Medium with high glucose (Gibco, Life Technologies Co, Carlsbad, CA, USA) supplemented with $1 \mathrm{mM}$ sodium pyruvate, $10 \%$ fetal bovine serum (FBS), and $1 \%$ penicillinstreptomycin (Gibco). The myeloid cell line 32D and 32D/ EGFR were cultured in Roswell Park Memorial Institute 1640 medium supplemented with $15 \%$ fetal bovine serum, 5\% WEHI 3B conditioned medium, $2 \mathrm{mM}$ L-glutamine, and $1 \%$ penicillin-streptomycin. The WEHI 3B cell line was maintained in Roswell Park Memorial Institute 1640 medium supplemented with $15 \%$ fetal bovine serum and $1 \%$ penicillin-streptomycin. WEHI $3 \mathrm{~B}$ conditioned medium containing interleukin-3 was produced by culturing WEHI 3B cells to a high density and then harvesting the supernatant.

\section{Surface modification of iron oxide nanoparticles with PEG}

SPIONs with a cross-linked dextran coating (nanomag ${ }^{\circledR}$ CLD-spio $100 \mathrm{~nm}$, Micromod Partikeltechnologie GmbH, 
Rostock, Germany), referred to as dexSPIONs in the following section, were functionalized with hydrazide via periodate oxidation. Briefly, $1 \mathrm{~mL}$ of $5 \mathrm{mg} / \mathrm{mL}$ dexSPIONs was incubated with $10 \mathrm{mM}$ periodate at room temperature for 30 minutes in the dark to allow partial oxidation of the dextran. The dihydrazide-PEG linker (molecular weight $5 \mathrm{kDa}$, Nanocs Inc, Boston, MA, USA) was added to the dexSPION solution. The resulting solution was incubated at room temperature for another 3 hours in the dark on a rolling shaker. The chemical reagents and excess dihydrazide-PEG linkers were removed by dialysis overnight at $4^{\circ} \mathrm{C}$ with a molecular weight cutoff of $100 \mathrm{kDa}$ Spectra/Por ${ }^{\circledR}$ FloatA-Lyzer ${ }^{\circledR}$ G2 dialysis device (Spectrum Laboratories Inc, Rancho Dominguez, CA, USA) in $50 \mathrm{mM}$ sodium acetate buffer ( $\mathrm{pH}$ 6.0). The hydrazone linkages were reduced to more stable secondary amine bonds by addition of $10 \mu \mathrm{L}$ of $5 \mathrm{M}$ sodium cyanoborohydride $\left(\mathrm{NaCNBH}_{3}\right)$ in $\mathrm{NaOH}$ per milliliter of conjugation mixture and incubation of the reaction overnight at $4^{\circ} \mathrm{C}$. The aldehyde sites that did not react were blocked for 30 minutes at room temperature by adding $50 \mu \mathrm{L}$ of $1.0 \mathrm{M}$ Tris- $\mathrm{HCl}$ blocking buffer per milliliter of conjugation solution. The reaction mixture was further purified using a MACS LS magnetic column attached to a high gradient magnet $\left(\mathrm{MACS}^{\circledR}\right.$ separation unit, Miltenyi Biotec GmbH, Bergisch Gladbach, Germany).

\section{Surface modification of iron oxide nanoparticles with cetuximab}

Functionalization of the PEG-dexSPIONs with cetuximab was achieved by adapting the periodate oxidation method. In brief, the carbohydrate moieties of cetuximab were oxidized by reaction with $10 \mathrm{mM}$ periodate at room temperature for 30 minutes in the dark. Oxidation was terminated by application of the reaction mixture to a PD MidiTrap G-25 desalting column (GE Healthcare, Piscataway, NJ, USA). Conjugation was achieved by addition of $1 \mathrm{mg}$ of hydrazide-functionalized PEG-dexSPIONs to $1 \mathrm{mg}$ of periodate-treated cetuximab monoclonal antibody and incubating the reaction in the dark on a rolling shaker for 4 hours at room temperature. After reduction by $\mathrm{NaCNBH}_{3}$ and blocking of the non-reacted aldehyde sites, the unconjugated cetuximab was removed using the MACS LS magnetic column to obtain the resulting cetuximab-functionalized iron oxide nanoparticles denoted as cet-PEG-dexSPIONs (Figure 1C).

\section{Binding affinity measurements by ELISA}

The binding affinity was determined by titrations of cetuximab, cet-PEG-dexSPIONs, or PEG-dexSPIONs on adsorbed EGFRvIII-ECD-hFc. After incubation at room temperature for 2 hours, bound primary antibody was detected with $100 \mu \mathrm{L}$ of horseradish peroxidase-conjugated goat antihuman kappa light chain antibody (Millipore, Bedford, MA, USA) diluted 1:10,000 in $100 \mu \mathrm{L}$ of blocking solution ( $1 \%$ BSA in $1 \times$ phosphate-buffered saline [PBS] containing $0.05 \%$ Tween-20). After one-hour incubation at room temperature, the enzymatic reaction was visualized by adding $100 \mu \mathrm{L}$ of NeA-Blue TMB substrate (Clinical Science Products Inc, Mansfield, MA, USA) to each well. The optical density was determined using a SpectraMax ${ }^{\circledR}$ M5 multi-mode microplate reader (Molecular Devices, Sunnyvale, CA, USA) at $450 \mathrm{~nm}$ after a 5-10-minute incubation at room temperature, followed by addition of $50 \mu \mathrm{L}$ of $2 \mathrm{~N} \mathrm{H}_{2} \mathrm{SO}_{4}$ to terminate the reaction. The relative affinities, expressed as $\mathrm{EC}_{50}$ values, were calculated using a four-parameter logistic equation with Sigmaplot ${ }^{\circledR}$ software (Systat Software Inc, San Jose, CA, USA).

\section{Immunocytochemical fluorescent staining}

A total of $6 \times 10^{4}$ cells were seeded in each well of an 8-well chamber slide (Millicell EZ slide, Millipore) and cultured overnight to allow adherence of the cells. The cells were fixed with $4 \%$ formaldehyde on ice for 20 minutes. After three washes, the cells were blocked with $0.2 \%(\mathrm{w} / \mathrm{v})$ BSA stain buffer (BD Bioscience Pharmingen, San Diego, CA, USA) at room temperature for one hour. The cells were incubated with $100 \mu \mathrm{L}$ of cetuximab and SPIONs diluted in $0.2 \%$ BSA stain buffer at the indicated concentrations on ice for one hour. Cells treated with $0.2 \%$ BSA stain buffer alone served as a negative control. After three washes, Dylight 488-conjugated goat $\mathrm{F}\left(\mathrm{ab}^{\prime}\right)_{2}$ anti-human $\mathrm{Fc} \gamma$ secondary antibody diluted in $0.2 \%$ BSA stain buffer was added to detect the bound cetuximab. After a one-hour incubation at room temperature, the cells were stained with 300 nM DAPI nucleic acid staining solution (Molecular Probes, Life Technologies Co) and rinsed several times with $1 \times$ PBS. After mounting with ProLong ${ }^{\circledR}$ Diamond antifade mountant (Molecular Probe), the slides were observed using a fluorescence microscope with the appropriate filters.

\section{Flow cytometry analysis}

To detect the binding of the synthesized SPIONs to the surface of A431 cells, indirect immunofluorescence staining was performed. In brief, $10^{6}$ cells were incubated on ice for one hour with cetuximab or the synthesized SPIONs diluted in $0.2 \%$ BSA stain buffer at the indicated concentrations. The cells were washed twice with cold $1 \times$ PBS to remove excess antibody and SPIONs, and subsequently incubated on ice in the dark for an additional one hour 
A

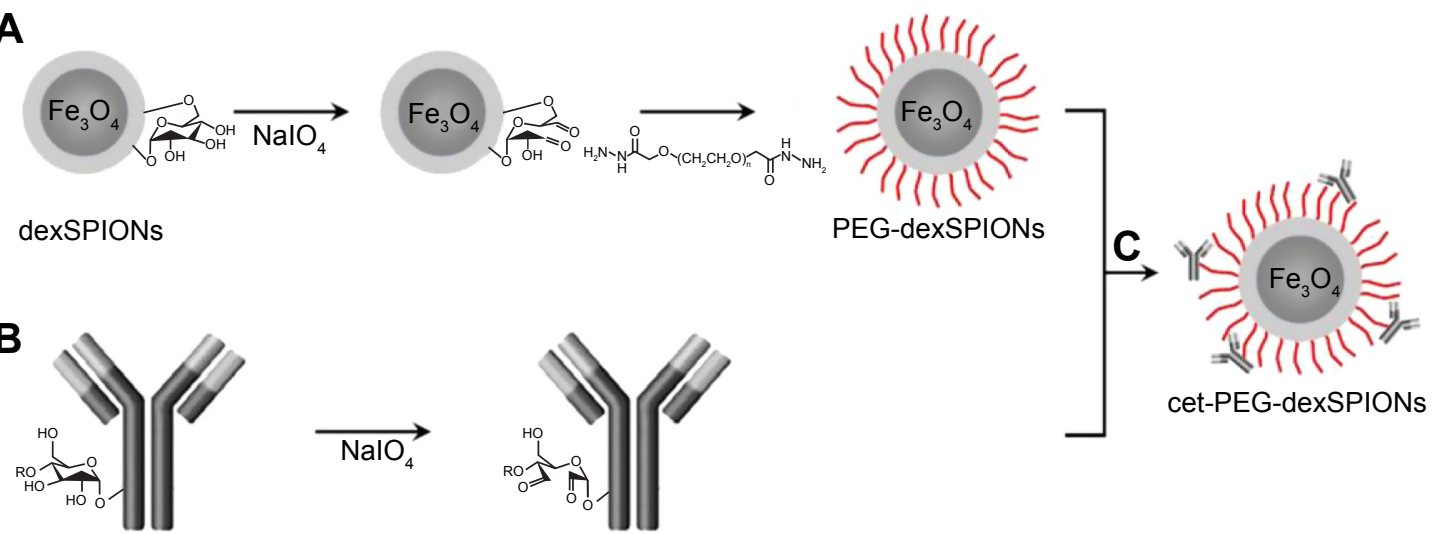

Figure I Bioconjugation scheme for cet-PEG-dexSPIONs.

Notes: (A) Surface modification of dextran-coated SPIONs with the dihydrazide-PEG linker (molecular weight, 5 kDa) via periodate oxidation of dextran. (B) Functionalization of cetuximab with active aldehyde functional groups via periodate oxidation. (C) Bioconjugation of cetuximab to PEG-dexSPIONs.

Abbreviations: cet, cetuximab; dex, dextran; PEG, polyethylene glycol; SPIONs, superparamagnetic iron oxide nanoparticles.

with R-phycoerythrin-conjugated mouse anti-human IgG monoclonal antibody (1:20; BD Biosciences Pharmingen) or fluorescein isothiocyanate (FITC)-conjugated mouse antidextran monoclonal antibody (1:5; StemCell Technologies Inc, Vancouver, BC, Canada) in $0.2 \%$ BSA stain buffer. The cells were washed twice, resuspended in cold $1 \times$ PBS, and analyzed immediately by flow cytometry (FACScan, Becton Dickinson, San Jose, CA, USA) to detect the intensity of cetuximab and SPIONs bound to the cell surface. Flow cytometry data were analyzed using Kaluza ${ }^{\circledR}$ flow cytometry analysis software (Beckman Coulter Inc, Brea, CA, USA).

The competition assay was performed by incubating A431 cells with cetuximab at the indicated concentrations prior to the treatment of $25 \mu \mathrm{g}$ Fe/mL cet-PEG-dexSPIONs. Fluorescein isothiocyanate (FITC)-conjugated mouse anti-dextran monoclonal antibody was added to quantify the membrane bound cet-PEG-dexSPIONs. In the specific binding assay for $10^{5} \mathrm{~A} 431$ cells spiked into $10^{6}$ human peripheral blood mononuclear cells, the cell mixture was pretreated with FcR blocking reagent (Miltenyi Biotec Inc) for 10 minutes at $4^{\circ} \mathrm{C}$ to block the binding of antibodies to $\mathrm{Fc}$ receptors on the surface of human peripheral blood mononuclear cells prior to treating the cell mixture with cetuximab or SPIONs. The signal intensity of the cell surface-bound cetuximab was detected using FITC-conjugated goat anti-mouse IgG secondary antibody.

\section{Cellular iron uptake assay}

After the cells were incubated with $25 \mu \mathrm{g} \mathrm{Fe} / \mathrm{mL}$ synthesized SPIONs for the indicated time, approximately $4 \times 10^{6}$ cells were washed three times with $1 \times$ PBS and dispensed in $100 \mu \mathrm{L}$ of $1 \times$ PBS. The samples were mineralized in $100 \mu \mathrm{L}$ of $12 \mathrm{~N} \mathrm{HCl}$ for 4 hours at room temperature. Next,
$400 \mu \mathrm{L}$ of $5 \%(\mathrm{w} / \mathrm{v})$ potassium thiocyanate was added to the solution. After a 15-minute incubation at room temperature, the suspension was centrifuged at $12,000 \times \mathrm{g}$ for 10 minutes, and $100 \mu \mathrm{L}$ of the supernatant was added to the wells of a 96-well plate. The iron concentration was determined by spectrophotometric measurements at $480 \mathrm{~nm}$ using a SpectraMax ${ }^{\circledR}$ M5 multi-mode microplate reader. The experiments were performed in four replicate wells. A calibration curve for the spectrophotometric determination of $\mathrm{Fe}^{3+}$ was prepared by evaluating known amounts of iron oxide nanoparticles.

\section{EGFR phosphorylation}

The A431 cells $\left(5 \times 10^{5}\right.$ cells per well) were grown overnight in culture medium in 6-well culture plates (Costar, Cambridge, MA, USA). The cells were starved in starvation medium (culture medium containing $0.5 \%$ fetal bovine serum) for 24 hours at $37^{\circ} \mathrm{C}$ and then treated under serum starvation conditions for 2 hours with cetuximab and the synthesized SPIONs prior to stimulation with $30 \mathrm{ng} / \mathrm{mL}$ epidermal growth factor for 30 minutes at $37^{\circ} \mathrm{C}$. The cells were harvested with a rubber scraper. After washing once with cold $1 \times$ PBS, the cell pellet was lysed with RIPA lysis buffer (1× PBS, $50 \mathrm{mM}$ Tris-HCl, $\mathrm{pH} 7.4,150 \mathrm{mM}$ $\mathrm{NaCl}, 1 \mathrm{mM}$ EGTA, $1 \mathrm{mM}$ sodium fluoride, $0.25 \%$ sodium deoxycholate, $1 \%$ NP-40, $1 \mathrm{mM}$ sodium orthovanadate, and $1 \mathrm{mM}$ phenylmethylsulfonyl fluoride) with $1 \%$ freshly added SigmaFAST ${ }^{\mathrm{TM}}$ protease inhibitor cocktail (s8830, Sigma-Aldrich, St Louis, MO, USA). The resulting mixture was centrifuged at $14,000 \times g$ for 10 minutes at $4^{\circ} \mathrm{C}$, and the protein concentration in the supernatant was measured using Bradford reagents (Sigma-Aldrich). Forty micrograms of total protein was then separated in a NuPAGE ${ }^{\circledR}$ Novex $^{\circledR}$ 
4\%-12\% Bis-Tris gel (Invitrogen, Life Technologies) and transferred to nitrocellulose membranes by electroblotting. The membranes were then incubated with primary antibodies against phospho-EGFR (Tyr1173; 1:2,000; Novus Biologicals Inc, Littleton, CO, USA) and $\beta$-actin (1:10,000; GeneTex Inc, Irvine, CA, USA), followed by horseradish peroxidaseconjugated goat anti-rabbit $\operatorname{IgG}(\mathrm{H}+\mathrm{L})$ secondary antibodies (1:10,000; Santa Cruz Biotechnology Inc, Santa Cruz, CA, USA). The bands were visualized with ECL Prime Western blotting detection reagent (Amersham Pharmacia Biotech Inc, Piscataway, NJ, USA) applied at a 1:1 ratio.

\section{EGFR internalization assay}

$5 \times 10^{5}$ A431 cells were incubated on ice for 10 minutes with cetuximab or the synthesized SPIONs diluted in serum-free medium at the indicated concentrations prior to incubation at $37^{\circ} \mathrm{C}$ for the indicated times to allow antibody-induced EGFR internalization. At each time point, the cells were washed once with ice-cold $1 \times$ PBS. After acid strip with ice-cold 0.2 $\mathrm{M}$ acetic acid and $0.5 \mathrm{M} \mathrm{NaCl}$ ( $\mathrm{pH} 2.5$ ), on ice for 5 minutes, the cells were washed once with ice-cold $1 \times$ PBS prior to incubation on ice for one hour with phycoerythrin-conjugated mouse anti-human EGFR monoclonal antibody (BD Bioscience Pharmingen) to label EGFR remained on the cell surface. Phycoerythrin-conjugated mouse IgG2b $\kappa$ isotype antibodies served as a negative control. Cells were washed twice, resuspended in ice-cold $1 \times \mathrm{PBS}$, and analyzed immediately using a FACScan flow cytometer (Becton Dickinson). Flow cytometry data were analyzed using CELLQuest ${ }^{\circledR}$ flow analysis software (Becton Dickinson).

\section{Apoptosis assay}

Apoptotic events were measured by double staining with Annexin V-FITC and propidium iodide according to the manufacturer's protocol (Annexin V-FITC apoptosis detection kit, eBioscience, San Diego, CA, USA). The cells were incubated at $37^{\circ} \mathrm{C}$ with culture medium containing cetuximab or the synthesized SPIONs for 24 hours as indicated. The cell populations were analyzed on a FACScan flow cytometer using the CELLQuest ${ }^{\circledR}$ flow analysis software.

\section{MRI phantom study}

The cells were incubated with PEG-dexSPIONs or cetPEG-dexSPIONs at iron concentrations of 10, 20, 40, 80, and $160 \mu \mathrm{g} / \mathrm{mL}$ for 2 hours at $37^{\circ} \mathrm{C}$. Cells treated with fresh culture medium alone served as a control. The washed cells were suspended in 1\% agarose gel for MRI using a $4.7 \mathrm{~T}$ Biospec 47/40 spectrometer (Bruker Inc, Ettlingen, Germany) equipped with a $72 \mathrm{~mm}$ volume coil as the radiofrequency transmitter and the receiver. The $\mathrm{T}_{2}$-weighted images were acquired with the following parameters: a fast spin-echo imaging sequence; a repetition time of 5,000 $\mathrm{msec}$; an echo time of $80 \mathrm{msec}$; a field of view of $6.2 \times 6.2 \mathrm{~cm}$; a matrix size of $256 \times 256$; four averages; and a slice thickness of $1.0 \mathrm{~mm}$.

\section{Results \\ Functionalization of dextran-coated SPIONs with cetuximab}

The procedure used to conjugate a therapeutic anti-EGFR monoclonal antibody, cetuximab, to a dextran-coated magnetic bead is illustrated in Figure 1. We used a dihydrazidePEG linker with a molecular weight of $5 \mathrm{kDa}$ to modify the surface of the dextran-coated SPIONs. The dextrans of the dexSPIONs were oxidized via periodate oxidation to create reactive aldehyde groups for spontaneous reaction with the hydrazide groups of the PEG linker to form hydrazone bonds, which were further reduced and stabilized with the mild reducing agent $\mathrm{NaCNBH}_{3}$. This process produced the PEGylated SPIONs, PEG-dexSPIONs, with hydrazide groups that were preserved on the free end of the PEG polymer chain for the following antibody immobilization step.

Using the same conjugation strategy as described, sugars in the polysaccharide groups of cetuximab were oxidized and then conjugated to the hydrazide groups on the surface of the PEG-dexSPIONs to form the antibody-functionalized SPIONs, cet-PEG-dexSPIONs. This coupling strategy enabled directional and covalent conjugation of antibody to iron oxide nanoparticles, with the antigen-binding sites directed outward from the particle surface, which is vital for maximizing the functional availability of the antibody for targeting.

\section{Characterization of the synthesized SPIONs}

The extent of antibody molecule conjugation to the magnetic beads was assessed by gel electrophoresis. As shown in Figure $2 \mathrm{~A}$, approximately $55 \%$ of the cetuximab was present as free antibody molecules (lane 3 ) in the antibody-SPION conjugation mixture after 4 hours of conjugation, as compared with the input amount of periodate-treated cetuximab (lane 1). In comparison with PEG-dexSPIONs (lane 2), a high molecular weight Coomassie blue-stained complex was trapped in the loading well of the polyacrylamide gel, suggesting that the antibody molecules were cross-linked to the PEG-dexSPIONs that have particle size larger than the gel pore size.

To further confirm that the high molecular weight complex was cet-PEG-dexSPIONs, we used agarose gel 

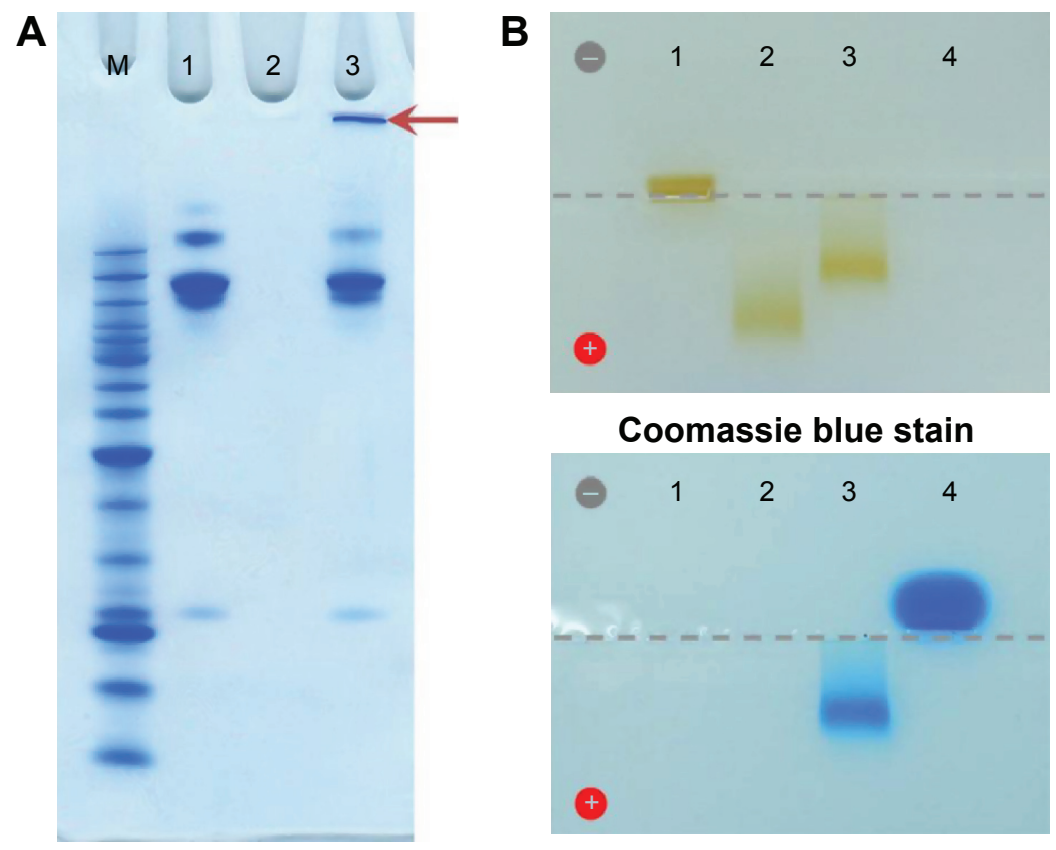

\section{Coomassie blue stain}

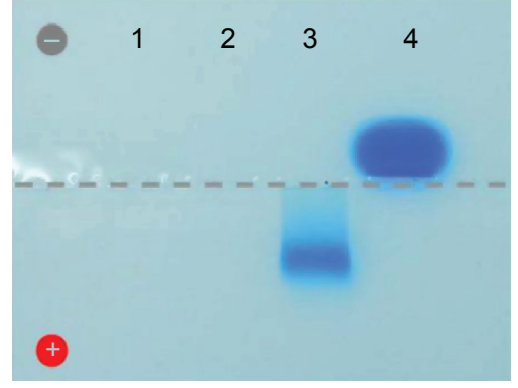

Figure 2 Confirmation of the bioconjugation of cetuximab to dexSPIONs by polyacrylamide gel and agarose gel electrophoresis analyses.

Notes: (A) Coomassie blue-stained 4\%-12\% Bis-Tris NuPAGE gel analysis revealed the successful cross-linking of cetuximab molecules on the surface of the iron oxide nanoparticles. Lane I, periodate-treated cetuximab; lane 2, PEG-dexSPIONs; lane 3, the cetuximab-SPION conjugation mixture incubated at room temperature for 4 hours. The arrow indicates the Coomassie blue-stained cetuximab-SPION conjugates, which were trapped in the loading well of the polyacrylamide electrophoresis gel due to their larger particle size. (B) The iron oxide nanoparticles were visible as a brown band with a differing mobility shift in the agarose gel. The antibody molecules were visualized by Coomassie blue staining. The sample loading well is indicated by a dotted gray line. Lane I, dexSPIONs; lane 2, PEG-dexSPIONs; lane 3, cet-PEG-dexSPIONs; lane 4, cetuximab. Abbreviations: cet, cetuximab; dex, dextran; PEG, polyethylene glycol; SPIONs, superparamagnetic iron oxide nanoparticles.

electrophoresis to analyze the various species before and after the conjugation reactions. As shown in Figure 2B, dexSPIONs, PEG-dexSPIONs, and cet-PEG-dexSPIONs were visualized as brown bands in the $0.65 \%$ agarose gel with a significantly different mobility shift toward the positive electrode. In TAE buffer at $\mathrm{pH} 8.0$, the cetuximab molecule, which has an isoelectric point at 8.48 , is positively charged and shifts in the opposite direction. Movement of the cetPEG-dexSPIONs toward the positive electrode seemed to be retarded due to conjugation of cetuximab to the surface of the PEG-dexSPIONs. The Coomassie blue-stained protein band representing the free cetuximab was diffuse in agarose gel. In contrast, for cet-PEG-dexSPIONs, the Coomassie blue-stained cetuximab colocalized with the iron oxide nanoparticles, indicating that cetuximab was chemically linked to the surface of the iron oxide nanoparticles.

Direct protein quantification in the presence of iron oxide nanoparticles was further performed to measure the average number of cetuximab molecules immobilized on one SPION. The amount of immobilized cetuximab on the surface of the magnetic nanoparticles was $3.496 \pm 0.294 \mu \mathrm{g}$ per $70 \mu \mathrm{g}$ Fe of cet-PEG-dexSPIONs based on the micro bicinchoninic acid protein assay and the thiocyanate-based spectrophotometric assay (Figure S1). We estimated that $6.56 \times 10^{12}$ dexSPIONs with a mean radius of $75.69 \mathrm{~nm}$ encapsulated approximately $1 \mathrm{mg}$ of iron. Therefore, the average number of immobilized cetuximab molecules per SPION was estimated to be $31 \pm 2$.

The average particle size estimated from the transmission electron microscopy images was approximately 10-20 nm for the three synthesized SPIONs. Figure S2 shows that the dexSPIONs maintained their morphology after surface modification with the PEG linker and further conjugation to cetuximab. No interparticle cross-linking occurred during the conjugation process. The mean hydrodynamic diameter determined by dynamic light scattering was $75.69 \pm 0.43 \mathrm{~nm}$ for the dexSPIONs, which increased to $112.73 \pm 0.74 \mathrm{~nm}$ after surface modification of the dexSPIONs with dihydrazidePEG linkers. After the PEG-dexSPIONs were further conjugated to cetuximab, the mean particle size decreased to $90.79 \pm 0.83 \mathrm{~nm}$ (Figure S3A and Table 1). Table 1 shows the zeta potential measurements at $\mathrm{pH} 7$ for these three colloidal suspensions. At $\mathrm{pH} 7$, the surface potential was $-7.3 \mathrm{mV}$ for the dexSPIONs, which is in good agreement with a previously reported value of $-9 \mathrm{mV}$ at $\mathrm{pH} 7$ for the dextran-coated iron oxide nanoparticles reported by Angeles et al. ${ }^{30}$ The zeta potential measured at $\mathrm{pH} 7$ was -29.2 and $-21.9 \mathrm{mV}$ for PEGdexSPIONs and cet-PEG-dexSPIONs, respectively. Different surface potential properties were observed for the particles, 
Table I Particle size and zeta potential of the synthesized iron oxide nanoparticles determined in double-distilled $\mathrm{H}_{2} \mathrm{O}$ using a Malvern-Zetasizer Nano instrument

\begin{tabular}{llll}
\hline Sample name & Particle size & $\begin{array}{l}\text { Zeta potential } \\
(\mathbf{m V})\end{array}$ \\
\cline { 2 - 4 } & $\begin{array}{l}\text { Z-average } \\
\text { (d.nm) }\end{array}$ & PdI & \\
\hline dexSPIONs & $75.69 \pm 0.43$ & $0.161 \pm 0.005$ & $-7.3 \mathrm{I}$ \\
PEG-dexSPIONs & $\mathrm{II} 2.73 \pm 0.74$ & $0.227 \pm 0.005$ & -29.2 \\
Cet-PEG-dexSPIONs & $90.79 \pm 0.83$ & $0.25 \mathrm{I} \pm 0.007$ & -21.9 \\
\hline
\end{tabular}

Abbreviations: cet, cetuximab; dex, dextran; Pdl, polydispersity index; PEG, polyethylene glycol; SPIONs, superparamagnetic iron oxide nanoparticles.

which indicated conjugation of cetuximab. The dexSPIONs were found to have high longitudinal $\left(r_{1}\right)$ and transverse $\left(r_{2}\right)$ relaxivity values $\left(22.575\right.$ and $162.8 \mathrm{mM}^{-1} \cdot \mathrm{s}^{-1}$, respectively), as measured using a Bruker Minispec at $20 \mathrm{mHz}$ (Figure S3B and Table 2). The surface-modified dexSPIONs with dihydrazide-PEG linkers caused a significant increase in the $r_{1}$ and $r_{2}$ relaxivity values $\left(29.18\right.$ and $252.25 \mathrm{mM}^{-1} \cdot \mathrm{s}^{-1}$, respectively), which dramatically decreased after the PEGdexSPIONs were further conjugated to cetuximab (10.586 and $110.08 \mathrm{mM}^{-1} \cdot \mathrm{s}^{-1}$, respectively). This result is consistent with previous reports demonstrating that conjugation of biomolecules onto the nanoparticle surface usually increases the overall thickness of the coating layer, thus decreasing the diffusion of the surrounding water molecules to the magnetic core, resulting in a reduced $r_{2}$ relaxivity. ${ }^{31}$

The $r_{2} / r_{1}$ relaxivity ratios for the synthesized SPION dispersions ranged from 7.21 to 10.4; these values are larger than that of the commercial $\mathrm{T}_{2}$ MRI contrast agent, Combidex ${ }^{\circledR}$, indicating that the synthesized SPIONs studied herein could serve as $T_{2}$ contrast agents, and with high efficacy. ${ }^{32}$

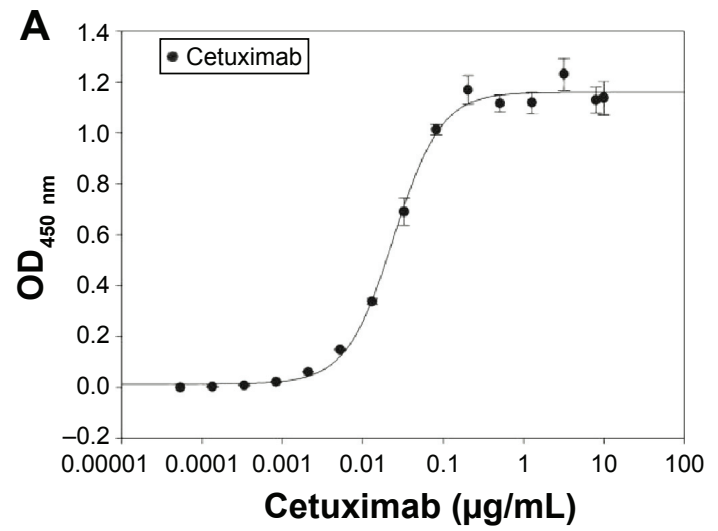

Table 2 Longitudinal and transversal relaxivities $\left(r_{1}\right.$ and $\left.r_{2}\right)$ of dexSPIONs and the synthesized nanoprobes at $20 \mathrm{mHz}$ at $37^{\circ} \mathrm{C}$ (Minispec)

\begin{tabular}{llll}
\hline Sample name & $\boldsymbol{r}_{\mathbf{1}}\left(\mathrm{mM}^{-1} \cdot \mathbf{s}^{-1}\right)$ & $r_{2}\left(\mathrm{mM}^{-1} \cdot \mathbf{s}^{-1}\right)$ & $r_{2} / r_{1}$ \\
\hline dexSPIONs & 22.575 & 162.8 & 7.21 \\
PEG-dexSPIONs & 29.18 & 252.25 & 8.64 \\
Cet-PEG-dexSPIONs & 10.586 & 110.08 & 10.40 \\
\hline
\end{tabular}

Note: Relaxivities shown were obtained in double-distilled water.

Abbreviations: cet, cetuximab; dex, dextran; PEG, polyethylene glycol; SPIONs, superparamagnetic iron oxide nanoparticles.

\section{Binding of cet-PEG-dexSPIONs to EGFRvIll and EGFR-overexpressing tumor cells}

An enzyme-linked immunosorbent assay (ELISA) was used to characterize the functional affinity of cetuximab after conjugation to PEG-dexSPIONs. Previous studies have confirmed the specific binding of cetuximab to EGFRvIII, the most common rearranged form of EGFR, by direct binding studies using ${ }^{125} \mathrm{I}$-labeled cetuximab. ${ }^{33}$ As shown in Figure 3, cetuximab as well as cet-PEG-dexSPIONs bound to surface-bound EGFRvIII-ECD-hFc protein in a concentration-dependent manner, whereas PEG-dexSPIONs had a negligible effect on the $\mathrm{OD}_{450}$ value, suggesting that the cetuximab molecule retained its binding activity after immobilization on the surface of PEG-dexSPIONs. The $\mathrm{EC}_{50}$ for cetuximab was estimated to be $0.0242 \mu \mathrm{g} / \mathrm{mL}$ (equal to $0.166 \mathrm{nM}$ ), with complete binding to the available EGFRvIII-ECD-hFc sites at concentrations $\geq 0.2 \mu \mathrm{g} / \mathrm{mL}$. By comparison, the $\mathrm{EC}_{50}$ for nanoparticle-conjugated cetuximab was $1.2938 \mu \mathrm{g} \mathrm{Fe} / \mathrm{mL}$, with complete binding to the available

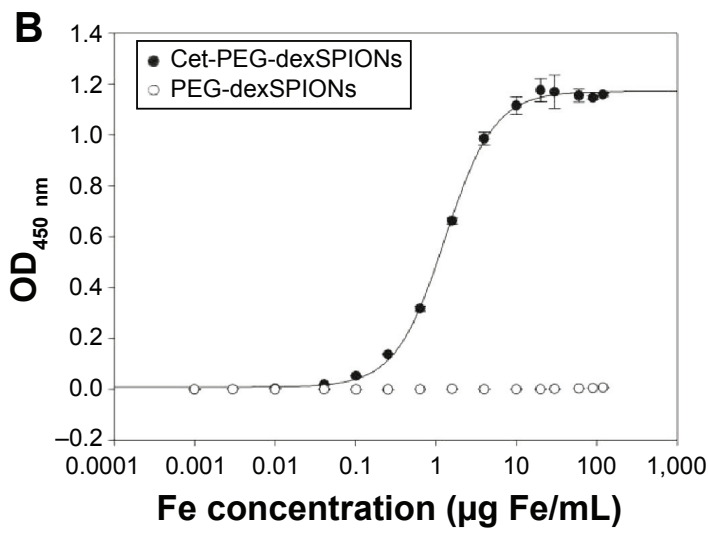

Figure 3 EGFRvIlI-ECD-hFc binding determined by enzyme-linked immunosorbent assay.

Notes: Soluble EGFRvIll-ECD-hFc was coated on enzyme-linked immunosorbent assay plates, and binding of cetuximab (A) or cetuximab-functionalized and non-functionalized iron oxide nanoparticles (B) was detected using a horseradish peroxidase-conjugated goat anti-human kappa light chain antibody. The concentrations tested ranged from $1.34 \times 10^{-4}$ to $10 \mu \mathrm{g} / \mathrm{mL}$ for the anti-EGFR monoclonal antibody cetuximab and from $6.4 \times 10^{-4}$ to $120 \mu \mathrm{g} \mathrm{Fe} / \mathrm{mL}$ for cet-PEG-dexSPIONs and PEG-dexSPIONs. The EC ${ }_{50}$ was 0.0242 and $0.0646 \mu \mathrm{g} / \mathrm{mL}$ for cetuximab and cet-PEG-dexSPIONs, respectively. Each point represents the mean \pm standard deviation of three determinations.

Abbreviations: cet, cetuximab; dex, dextran; ECD, extracellular domain; EGFR, epithelial growth factor receptor; OD, optical density; PEG, polyethylene glycol; SPIONs, superparamagnetic iron oxide nanoparticles. 
EGFRvIII-ECD-hFc sites at concentrations $\geq 10 \mu \mathrm{g} \mathrm{Fe} / \mathrm{mL}$. Based on our protein quantification results, this $\mathrm{EC}_{50}$ value was $0.0646 \mu \mathrm{g} / \mathrm{mL}$ (equivalent to $0.443 \mathrm{nM}$ ) in terms of the antibody concentration. The ELISA results revealed a slightly diminished binding affinity of the nanoparticle-conjugated cetuximab compared with the free form of the antibody. The decrease in binding affinity might have been caused by a steric hindrance effect.

To further characterize the binding specificity of the cet-PEG-dexSPIONs toward EGFR-expressing tumor cells, immunofluorescence was performed using the A431 human epidermoid carcinoma cell line. A431 cells have been reported to express high levels of EGFR (approximately $3.51 \times 10^{6}$ EGFR molecules per cell). ${ }^{23}$ Indirect immunostaining with Dylight 488-conjugated goat $\mathrm{F}\left(\mathrm{ab}^{\prime}\right)_{2}$ anti-human $\mathrm{Fc} \gamma$ secondary antibody was performed to visualize the presence of cetuximab attached to the cell surface after incubating the A431 cells on ice for one hour with cetuximab or the synthesized SPIONs at the indicated concentrations. There was a green color on the cell membrane of A431 cells incubated with cetuximab and cet-PEG-dexSPIONs but not on their nonfunctional counterparts (Figure 4A). No signal was detected on the cell membrane of EGFR-negative Roswell Park Memorial Institute 2650 cells in any of the treatments (data not shown).

To evaluate the ability of the cet-PEG-dexSPIONs to target A431 cells, R-phycoerythrin-conjugated mouse antihuman IgG monoclonal antibody was used to reveal the binding of cetuximab with its cargo to the cell surface via flow cytometry analysis. Figure 4B shows the targeted binding of cet-PEG-dexSPIONs to A431 cells. No fluorescence signal was detected in A431 cells that were co-incubated with human IgG (negative control) or human IgG-conjugated PEG-dexSPIONs (data not shown). In contrast, the binding of cet-PEG-dexSPIONs and cetuximab (positive control) to A431 cells resulted in a significant increase in fluorescence intensity. Moreover, the median fluorescence intensity increased with increasing doses of cet-PEG-dexSPIONs, illustrating dose-dependent binding activity to A431 cells. To further quantify the amount of iron oxide nanoparticles attached to the surface of target cells exposed to the same treatment, FITC-conjugated mouse anti-dextran monoclonal antibody was used to stain the dextran coat of the synthesized SPIONs (Figure 4C). No dextran signal was detected on the surface of A431 cells incubated with human IgG, cetuximab, PEG-dexSPIONs, or human IgG-conjugated PEG-dexSPIONs (data not shown), whereas there was a significant dose-dependent increase in the dextran signal in
A431 cells co-incubated with cet-PEG-dexSPIONs. This result indicates that conjugation of cetuximab enhances the binding of iron oxide nanoparticles to the surface of EGFR-expressing tumor cells. The competitive binding study revealed that cetuximab efficiently blocked the surface EGFR sites and prevented binding of cet-PEG-dexSPIONs (Figure 4D). This finding demonstrated the specificity of the synthesized SPIONs for EGFR-expressing tumor cells. To confirm the specific binding of cet-PEG-dexSPIONs to target cells, flow cytometry analysis was performed using a mixture of non-target peripheral blood mononuclear cells and A431 target cells at a ratio of 10 to 1 . Figure 4E shows that the specificity of cetuximab and cet-PEG-dexSPIONs toward A431 cells was not interfered with by the presence of peripheral blood mononuclear cells.

The in vitro cytotoxic effects of PEG-dexSPIONs and cet-PEG-dexSPIONs was evaluated in human foreskin fibroblast (Hs68) cell line using the Alamar Blue cytotoxicity assay. The viability of untreated cells was used as a control. After different periods of incubation, the viability of each sample was above $90 \%$ in relation to the control cells, indicating that these synthesized SPIONs have low cytotoxicity, even at an iron concentration up to $100 \mu \mathrm{g} / \mathrm{mL}$ (Figure S4). EGFR expression levels on the cell surface were determined by flow cytometry. Direct immunofluorescence staining of the tumor cell lines showed that A431 and 32D/ EGFR cells express high levels of EGFR on their surface, whereas no EGFR expression was detected on ErbB-null 32D cells (Figure S5). To evaluate the selectivity of cet-PEGdexSPIONs, the iron content of $4 \times 10^{6}$ of these differential EGFR-expressing tumor cells was measured after 2 hours of incubation at $37^{\circ} \mathrm{C}$ with $25 \mu \mathrm{g} \mathrm{Fe} / \mathrm{mL}$ of the PEG-dexSPIONs or cet-PEG-dexSPIONs, as seen in Table 3 and Figure 5. The results revealed almost the same endogenous iron content in the three tumor cell lines at a cell number of $4 \times 10^{6}:$ ie, $0.330 \pm 0.0033,0.335 \pm 0.0039$, and $0.344 \pm 0.0057 \mu \mathrm{g} \mathrm{Fe} / 4 \times 10^{6}$ cells for 32D, 32D/EGFR, and A431 cells, respectively. Meanwhile, the intracellular iron content after incubation with PEG-dexSPIONs only increased slightly, demonstrating a fold increase of 2.02, 2.38, and 1.60 for 32D, 32D/EGFR and A431 cells, respectively, compared with the corresponding untreated control cells.

A significant increase in iron concentration was observed in A431 cells and 32D/EGFR cells compared with EGFR-null $32 \mathrm{D}$ cells after incubation with cet-PEG-dexSPIONs. There was a fold increase of 18.61 and 8.27 for A431 and 32D/ EGFR cells, respectively. However, functionalization of the magnetic nanoprobes with cetuximab did not increase iron 
A

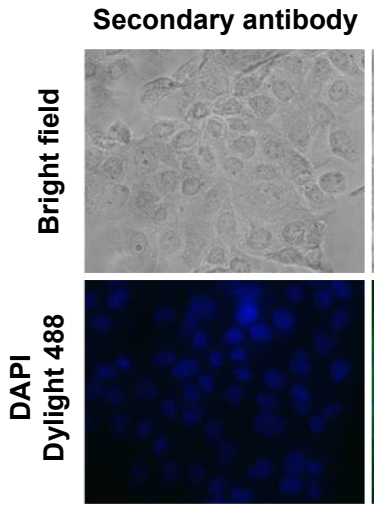

B

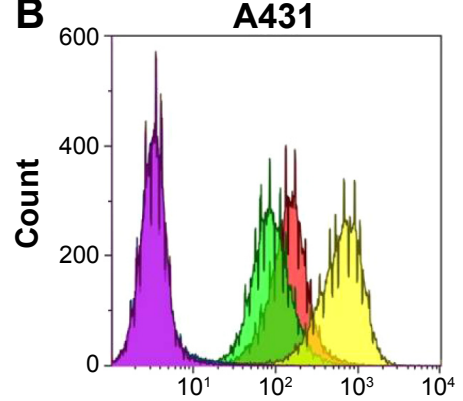

Human IgG intensity

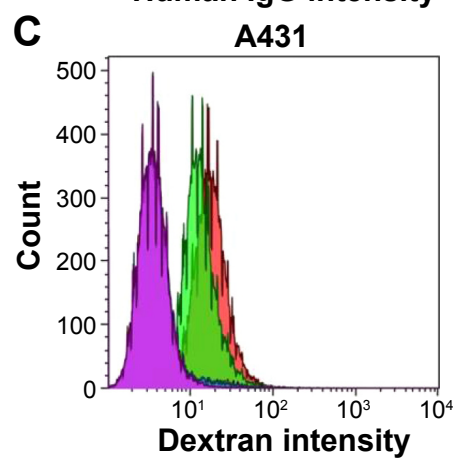

D

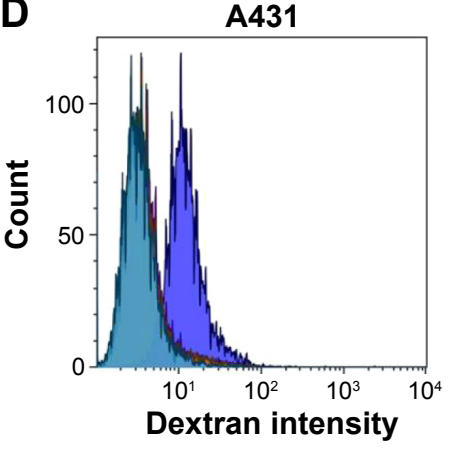

Cetuximab
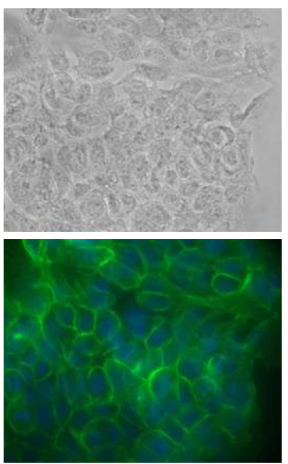

PEG-dexSPIONs
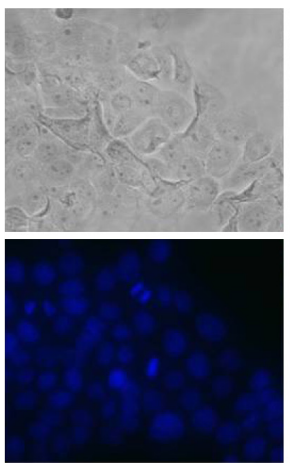

\section{Cet-PEG-dexSPIONs}
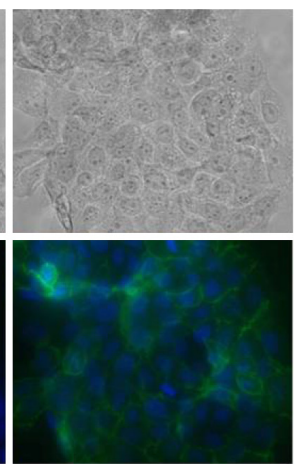

E

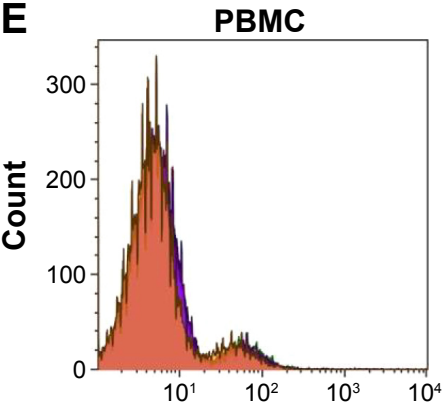

Mouse IgG intensity

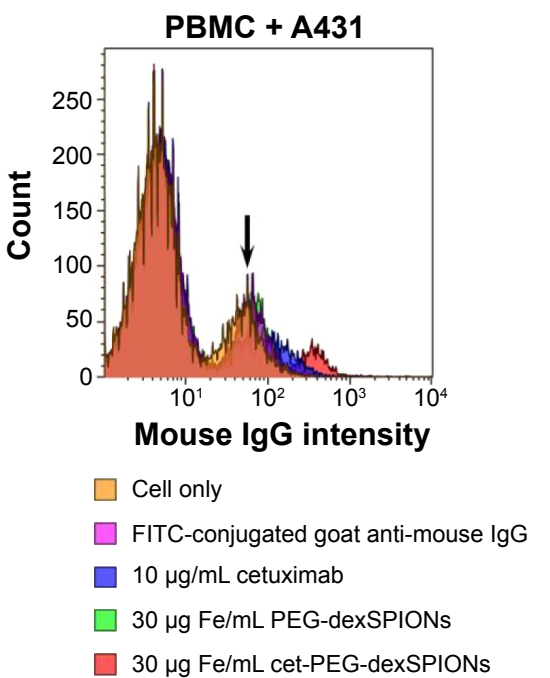

$30 \mu \mathrm{g} \mathrm{Fe} / \mathrm{mL}$ cet-PEG-dexSPIONs
A431 cell only

PE-conjugated mouse anti-human IgG mAb

$10 \mu \mathrm{g} / \mathrm{mL}$ human $\mathrm{IgG}$

$10 \mu \mathrm{g} / \mathrm{mL}$ cetuximab

$25 \mu \mathrm{g} \mathrm{Fe} / \mathrm{mL}$ PEG-dexSPIONs

$25 \mu \mathrm{g} \mathrm{Fe} / \mathrm{mL}$ cet-PEG-dexSPIONs

$\square 50 \mu \mathrm{g} \mathrm{Fe} / \mathrm{mL}$ cet-PEG-dexSPIONs

\section{$\square$ A431 cell only}

FITC-conjugated mouse anti-dextran mAb

$\square 10 \mu \mathrm{g} / \mathrm{mL}$ human $\operatorname{lgG}$

$\square 10 \mu \mathrm{g} / \mathrm{mL}$ cetuximab

$25 \mu \mathrm{g} \mathrm{Fe} / \mathrm{mL}$ PEG-dexSPIONs

$\square 25 \mu \mathrm{g} \mathrm{Fe} / \mathrm{mL}$ cet-PEG-dexSPIONs

$\square 50 \mu \mathrm{g} \mathrm{Fe} / \mathrm{mL}$ cet-PEG-dexSPIONs

\section{$\square$ A431 cell only}

$\square$ FITC-conjugated mouse anti-dextran mAb

$25 \mu \mathrm{g} / \mathrm{mL}$ PEG-dexSPIONs

$25 \mu \mathrm{g} / \mathrm{mL}$ cet-PEG-dexSPIONs

$10 \mu \mathrm{g} / \mathrm{mL}$ cetuximab $+25 \mu \mathrm{g} / \mathrm{mL}$ cet-PEG-dexSPIONs

$20 \mu \mathrm{g} / \mathrm{mL}$ cetuximab $+25 \mu \mathrm{g} / \mathrm{mL}$ cet-PEG-dexSPIONs

Figure 4 Binding specificity of cetuximab, PEG-dexSPIONs, and cet-PEG-dexSPIONs.

Notes: (A) For immunostaining of A43I cells, the cells were incubated with the indicated antibody or particles on ice for one hour. Dylight 488-conjugated anti-human Fc $\gamma$ antibody was used as the detection antibody for fluorescence imaging. The nuclei of the cells were counterstained with DAPI (blue color). Bright field and merged cell images of Dylight 488/DAPI are shown in the upper and bottom rows, respectively. Images were obtained with a 40x objective lens. (B, C) Flow cytometry analysis of antibodies or nanoparticles specific to A43I cells. A43I cells were incubated with human IgG (negative control), cetuximab (positive control), or the synthesized magnetic probes at the indicated concentrations on ice for one hour. The cells were then stained on ice in the dark with R-PE-conjugated mouse anti-human lgG $\mathrm{mAb}$ (B) and fluorescein isothiocyanate-conjugated mouse anti-dextran $\mathrm{mAb}$ (C) for one hour to detect the human $\operatorname{lgG}$ molecules and dextran-coated magnetic nanoparticles, respectively, on the surface of A43I cells. (D) Competition assay by flow cytometry. The competition assay was performed as described in (C) except that A43I cells were treated on ice for one hour with 10 or $20 \mu \mathrm{g} / \mathrm{mL}$ cetuximab prior to treatment with the iron oxide nanoparticles. (E) Flow cytometry results showing that cet-PEG-dexSPIONs specifically targeted A43I that was spiked into PBMCs ( $10^{5} \mathrm{~A} 43 \mathrm{I}$ cells were spiked into $\left.10^{6} \mathrm{PBMCs}\right)$. Fc receptors on the surface of PBMCs were blocked with FcR blocking reagents at $4^{\circ} \mathrm{C}$ for 20 minutes, and the cells were then incubated with cetuximab (positive control) or the synthesized magnetic probes at the indicated concentrations on ice for one hour. The cells were then immediately stained on ice in the dark with fluorescein isothiocyanate-conjugated goat anti-mouse $\operatorname{lgG}$ secondary antibody for an additional 30 minutes to detect cetuximab molecule binding to the surface of A43I cells. The arrow indicates the signal for unstained A43I cells.

Abbreviations: cet, cetuximab; DAPI, 4',6-diamidino-2-phenylindole; dex, dextran; Ig, immunoglobulin; PEG, polyethylene glycol; SPIONs, superparamagnetic iron oxide nanoparticles; PBMCs, peripheral blood mononuclear cells; PE, phycoerythrin; mAb, monoclonal antibody. 
Table 3 Iron content of differential EGFR-expressing cells after a 2-hour incubation at $37^{\circ} \mathrm{C}$ with the synthesized iron oxide nanoparticles

\begin{tabular}{|c|c|c|c|c|}
\hline Cell line & Treatment & $\begin{array}{l}\text { Mean iron concentration } \\
\left(\mu \mathrm{g} \mathrm{Fe} / 4 \times 10^{6} \text { cells }\right)\end{array}$ & SD & Fold \\
\hline \multirow[t]{3}{*}{$32 \mathrm{D}$} & Cell only & 0.330 & 0.0033 & 1.00 \\
\hline & PEG-dexSPIONs & 0.667 & 0.0093 & 2.02 \\
\hline & Cet-PEG-dexSPIONs & 0.772 & 0.0092 & 2.34 \\
\hline \multirow[t]{3}{*}{ 32D/EGFR } & Cell only & 0.335 & 0.0039 & 1.00 \\
\hline & PEG-dexSPIONs & 0.798 & 0.0081 & 2.38 \\
\hline & Cet-PEG-dexSPIONs & 2.771 & 0.0111 & 8.27 \\
\hline \multirow[t]{3}{*}{ A43I } & Cell only & 0.344 & 0.0057 & 1.00 \\
\hline & PEG-dexSPIONs & 0.549 & 0.0022 & 1.60 \\
\hline & Cet-PEG-dexSPIONs & 6.401 & 0.0937 & $|8.6|$ \\
\hline
\end{tabular}

Abbreviations: cet, cetuximab; dex, dextran; EGFR, epidermal growth factor receptor; PEG, polyethylene glycol; SD, standard deviation; SPIONs, superparamagnetic iron oxide nanoparticles.

uptake in 32D cells. These results confirm that the engineered targeted magnetic nanoprobes were capable of actively targeting EGFR-expressing tumor cells and could also differentiate tumor cell lines with differential EGFR expression levels.

\section{Therapeutic efficacy of cet-PEG- dexSPIONs}

To assess the ability of cet-PEG-dexSPIONs to inhibit epidermal growth factor-induced EGFR phosphorylation by preventing epidermal growth factor binding, 24-hour

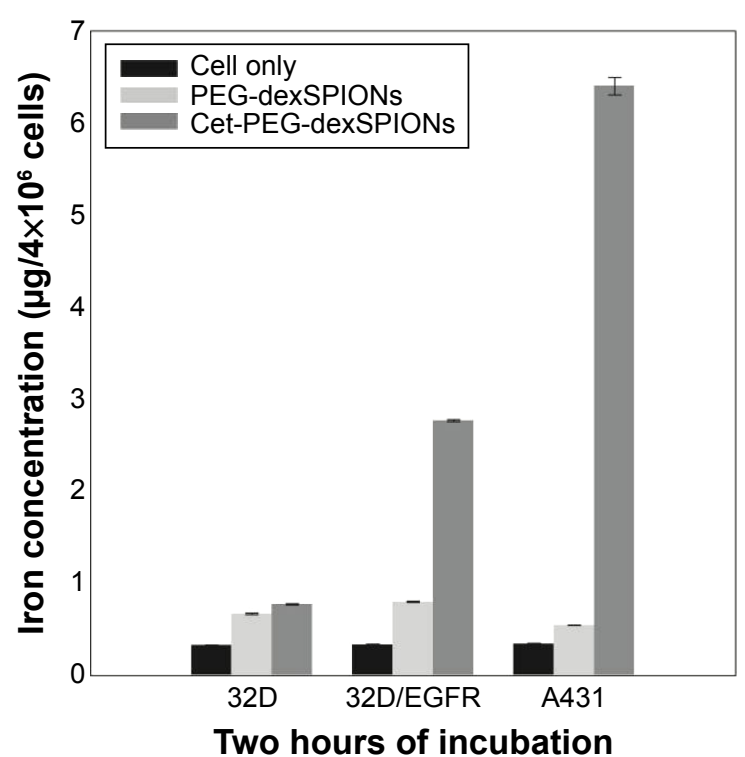

Figure 5 Iron uptake among the cell lines with differential EGFR expression levels. The A43I, 32D, and 32D/EGFR cell lines were incubated with $25 \mu \mathrm{g} \mathrm{Fe} / \mathrm{mL}$ of PEG-dexSPIONs or cet-PEG-dexSPIONs at $37^{\circ} \mathrm{C}$ for 2 hours. The excess iron was removed by washing with I $\times$ phosphate-buffered saline, and the iron concentration in $4 \times 10^{6}$ cells from each cell line was determined using a thiocyanate-based spectrophotometric assay. The results are presented as the mean \pm standard deviation of four determinations.

Abbreviations: cet, cetuximab; dex, dextran; EGFR, epidermal growth factor receptor; PEG, polyethylene glycol; SPIONs, superparamagnetic iron oxide nanoparticles. serum-starved A431 cells were treated with varying doses of the synthesized SPIONs at $37^{\circ} \mathrm{C}$ for 2 hours prior to stimulation with or without epidermal growth factor for 30 minutes. There was slight phosphorylation of EGFR in the serum starvation-treated A431 control cells not treated with epidermal growth factor (Figure 6A). As expected, phosphorylation of EGFR was enhanced by treatment with epidermal growth factor, and this enhancement of phosphorylation was inhibited when the cells were preincubated with cetuximab. Although there was a high level of EGFR phosphorylation in A431 cells pretreated with PEG-dexSPIONs, we observed a dose-dependent inhibition of epidermal growth factorinduced EGFR phosphorylation in response to pretreatment of A431 cells with cet-PEG-dexSPIONs, with an almost complete inhibition of phosphorylation at an iron concentration of $50 \mu \mathrm{g} / \mathrm{mL}$ cet-PEG-dexSPIONs.

Our results also revealed a slight decrease in cellular EGFR expression following pretreatment with cetuximab. This result is in agreement with previous findings demonstrating that treatment of cells with cetuximab resulted in EGFR internalization, downregulation of membrane EGFR levels, and a reduction in cellular EGFR protein levels. ${ }^{12}$ Interestingly, pretreatment with cet-PEG-dexSPIONs provoked a more significant decrease in expression of cellular EGFR protein compared with cetuximab alone, as shown by the almost complete elimination of cellular EGFR protein in A431 cells that were preincubated with $50 \mu \mathrm{g} / \mathrm{mL}$ cet-PEG-dexSPIONs. Nanoconjugation has been reported to enhance cetuximabinduced EGFR endocytosis and to influence intracellular trafficking of EGFR in pancreatic cancer cells. ${ }^{34}$ To examine the ability of cet-PEG-dexSPIONs to induce degradation of EGFR by enhancing internalization of the receptor, the kinetics of cet-PEG-dexSPION-induced EGFR downregulation were compared with those of cetuximab-induced EGFR 

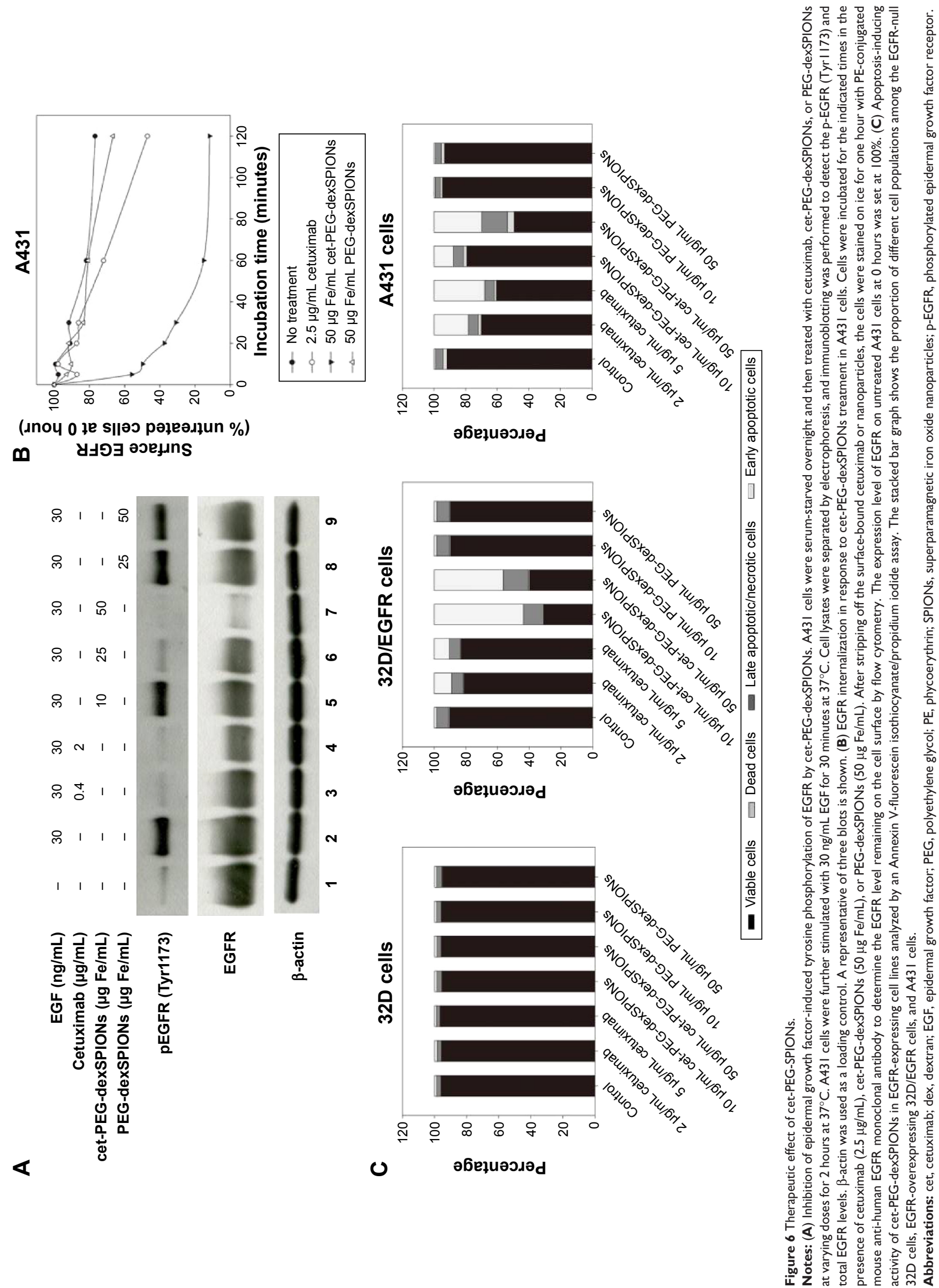
downregulation using flow cytometry following treatments for different incubation times at $37^{\circ} \mathrm{C}$. As shown in Figure $6 \mathrm{~B}$, we observed a slight decrement in surface EGFR expression in PEG-dexSPION-treated A431 cells within 2 hours of incubation at $37^{\circ} \mathrm{C}$, which is comparable with the results obtained for untreated cells. The maximum surface EGFR downregulation was $23.3 \%$ and $33.4 \%$ for untreated and PEGdexSPION-treated A431 cells, respectively. Treatment with cet-PEG-dexSPIONs resulted in a reduction of more than $40 \%$ in the level of surface EGFR within 5 minutes compared with a $13 \%$ reduction upon treatment with an equivalent amount of cetuximab. The surface EGFR decreased significantly with increasing incubation time, with a maximum EGFR surface downregulation of $88.3 \%$ in response to treatment with cet-PEG-dexSPIONs after 2 hours. However, a maximum reduction in surface EGFR of only $53 \%$ was observed in response to treatment with cetuximab. These data indicate that cet-PEG-dexSPIONs mediated EGFR internalization much more efficiently than cetuximab. These data, together with almost complete degradation of the total EGFR expression level observed in our Western blot analysis, indicate that the EGFR cross-linking ability of cet-PEG-dexSPIONs resulting from conjugation of cetuximab molecules to one iron oxide nanoparticle might accelerate EGFR endocytosis and degradation of cellular EGFR protein by lysosomal enzymes.

To further investigate the ability of cet-PEG-dexSPIONs to induce apoptosis in EGFR-expressing tumor cell lines, we treated A431, 32D and 32D/EGFR cells with different doses of cetuximab, cet-PEG-dexSPIONs, or PEG-dexSPIONs for 24 hours. Apoptosis was evaluated using the Annexin V-FITC/ propidium iodide double staining kit (Figures 6C and S6). No apoptosis or cell death was observed in $32 \mathrm{D}$ cells after a 24-hour treatment with increasing doses of cetuximab. In comparison, there was a significant increase in the early apoptotic cell population and slight increases in the late apoptotic/ necrotic cell populations in the cetuximab-treated A431 cell lines, while a slight increase in the early apoptotic cell population was detected in the cetuximab-treated 32D/EGFR cells. Treatment with cet-PEG-dexSPIONs induced apoptosis in a dose-dependent manner in A431 cells. Significant apoptotic events also occurred in cet-PEG-dexSPION-treated 32D/ EGFR cells. After treating A431 and 32D/EGFR cells with $5 \mu \mathrm{g} / \mathrm{mL}$ cetuximab, $39.41 \%$ and $17.01 \%$ of cells, respectively, were nonviable. Here, nonviable cells are defined as cells that appeared in the first, second and fourth quadrants of the flow cytometry graphs in Figure S6. Upon treatment of cet-PEG-dexSPIONs containing only half the amount of cetuximab, the percentages of nonviable cells increased to
$50.61 \%$ and $60.11 \%$, respectively. Interestingly, we also observed a clear increase in the late apoptotic/necrotic cell population in A431 cells treated with $50 \mu \mathrm{g}$ Fe/mL cet-PEGdexSPIONs and in 32D/EGFR cells treated with 10 and $50 \mu \mathrm{g} \mathrm{Fe} / \mathrm{mL}$ cet-PEG-dexSPIONs. These results indicate that cet-PEG-dexSPIONs greatly accelerated the apoptotic process. No apoptosis was induced in the cell lines treated with PEG-dexSPIONs at any concentration.

Upon binding to the EGFR expressed on the surface of tumor cells, the antitumor activity of cetuximab was dependent at least in part on subsequent binding of natural killer cells to the Fc portion of the antibody, with consequent destruction of tumor cells via an ADCC mechanism. To identify the optimal cetuximab concentration for the ADCC assay, the lactate dehydrogenase released from the cytosol of damaged tumor cells was measured after a 4-hour incubation with cetuximab concentrations ranging from 0.00001 to $10 \mu \mathrm{g} / \mathrm{mL}$ using an E:T ratio of $10: 1$. NK-92 natural killer cells, which are modified to express the high affinity variant of CD16 (FcyRIIIA) on the cell surface, ${ }^{35}$ were used as effector cells. A cetuximab concentration in excess of $0.1 \mu \mathrm{g} / \mathrm{mL}$ was sufficient for maximum ADCC activity against A431 cells with high expression of EGFR at an E:T ratio of 10:1 (Figure S7A). Therefore, we used cetuximab at a concentration of $0.5 \mu \mathrm{g} / \mathrm{mL}$ as a standard to test whether cet-PEG-dexSPIONs retain the ability to induce ADCC activity in EGFR-expressing tumor cell lines. Cetuximab at a concentration of $0.5 \mu \mathrm{g} / \mathrm{mL}$ showed enhanced target cell lysis of approximately $40.5 \%$ above the background level due to natural killer cell-mediated cytotoxicity. In contrast, approximately $10.4 \%$ of the A431 target cells were lysed by $10 \mu \mathrm{g}$ of $\mathrm{Fe} / \mathrm{mL}$ cet-PEG-dexSPIONs, which contain an equivalent amount of cetuximab on their surface (Figure S7B). No ADCC activity against A431 cells was detected for PEGdexSPIONs. These data indicate that cet-PEG-dexSPIONs could mediate the effect of ADCC upon binding to EGFR, demonstrating that the therapeutic activity of cetuximab was preserved after immobilization onto PEG-dexSPIONs.

\section{MRI phantom study}

The MRI signal sensitivity of the synthesized SPIONs was investigated by labeling the cells in vitro and performing MRI of the labeled cells suspended in agarose gel. No $\mathrm{T}_{2}$ hypointense signal was detected on MRI of the unlabeled cells. Remarkably, when cet-PEG-dexSPIONs were applied, we observed a significant negative enhancement of the EGFRexpressing tumor cells in comparison with PEG-dexSPIONs (Figure 7). On the $\mathrm{T}_{2}$-weighted MRI scans, while the reduction 

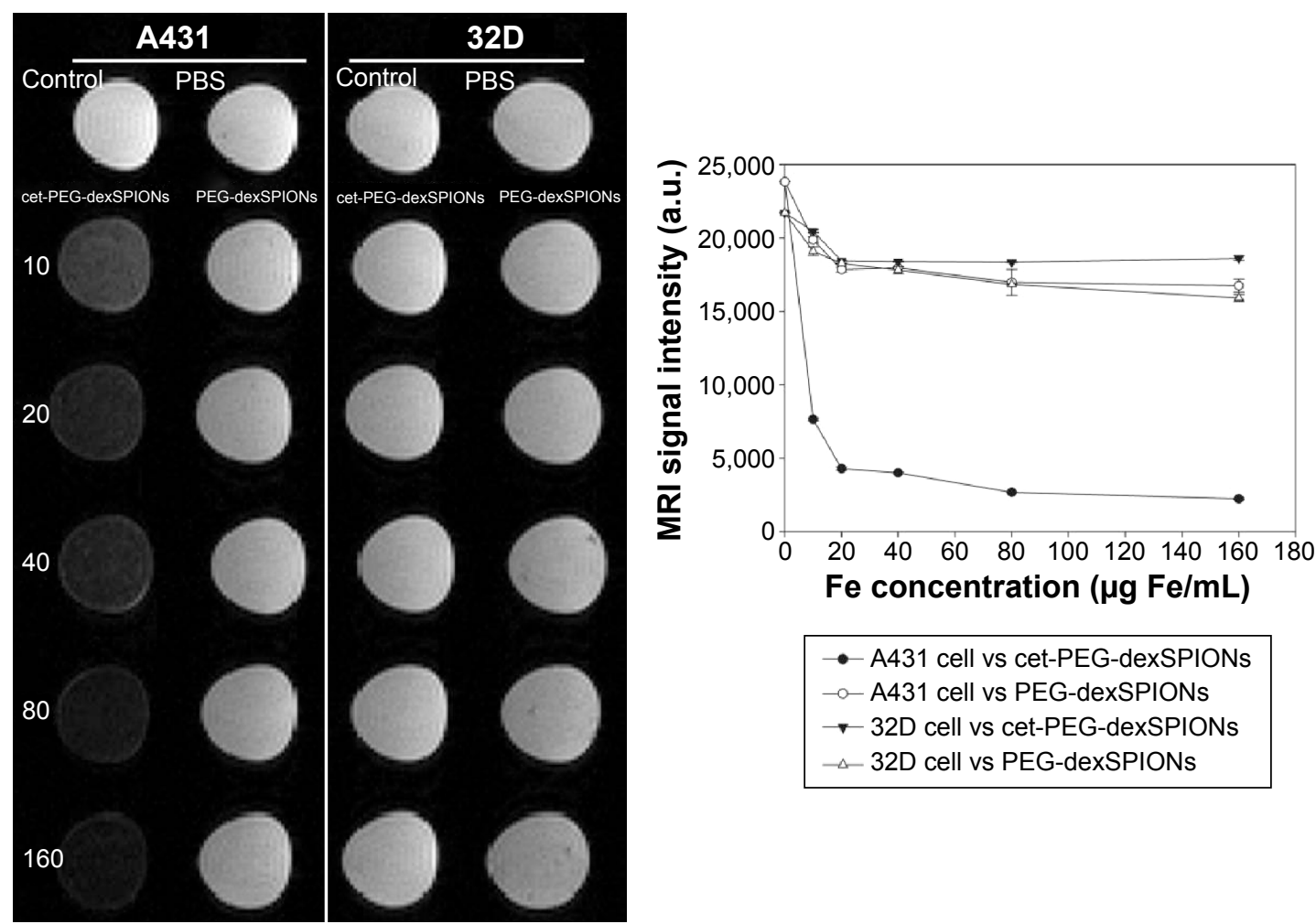

- A431 cell vs cet-PEG-dexSPIONs

- $\mathrm{A} 431$ cell vs PEG-dexSPIONs

- $32 \mathrm{D}$ cell vs cet-PEG-dexSPIONs

$\triangle-32 D$ cell vs PEG-dexSPIONs

Figure $7 \mathrm{~T}_{2}$-weighted image of A43I and 32D cells at different doses of the synthesized SPIONs. A43I and 32D cells were incubated at $37^{\circ} \mathrm{C}$ for 2 hours with PEG-dexSPIONs or cet-PEG-dexSPIONs at iron concentrations of $10,20,40,80$, and $160 \mu \mathrm{g} / \mathrm{mL}$. Tumor cells treated with fresh culture medium alone served as a control. The excess iron was removed by washing with IXPBS, and the cells were suspended in a I\% agarose gel for the following in vitro MRI analysis using a $4.7 \mathrm{~T}$ scanner. The agarose gel containing PBS alone without any cells was used as a blank. The plot of the MRI signal intensity as a function of the iron concentration of the synthesized SPIONs revealed the MRI capability of the synthesized magnetic nanoparticles.

Abbreviations: cet, cetuximab; dex, dextran; MRI, magnetic resonance imaging; PEG, polyethylene glycol; PBS, phosphate-buffered saline; SPIONs, superparamagnetic iron oxide nanoparticles; a.u., arbitrary units.

in signal intensity generated by PEG-dexSPIONs was $25.1 \%$ and $15.9 \%$ in the A431 and 32D cell lines, respectively, cetPEG-dexSPIONs reduced the MRI intensity by $81.9 \%$ in the A431 cell line compared with a $15.1 \%$ reduction in the $32 \mathrm{D}$ cell line at an iron concentration of $20 \mu \mathrm{g} / \mathrm{mL}$. This result indicates a specific and active targeting effect of cet-PEG-dexSPIONs to A431 cells with high EGFR expression, which showed highcontrast enhancement on $\mathrm{T}_{2}$-weighted MRI images.

Statistically significant differences in signal intensity were sought by analysis of variance followed by Tukey's multiple range test. The analysis revealed a statistically significant signal intensity difference on $\mathrm{T}_{2}$-weighted MRI between PEG-dexSPIONs and cet-PEG-dexSPIONs at the same iron concentration in A431 cells $(P<0.001)$. These phantom MRI images indicate that cet-PEG-dexSPIONs represent a sensitive $T_{2}$-weighted MRI contrast agent for targeting EGFR-expressing tumor cells.

\section{Discussion}

In the present study, we designed a method for conjugating a therapeutic antibody to a molecular imaging nanoparticle for targeted treatment of tumor cells while simultaneously providing enhanced contrast on $\mathrm{T}_{2}$-weighted MRI. Our in vitro assay results indicate that cet-PEG-dexSPIONs could be developed as a promising theranostic nanomedicine for therapeutic targeting of EGFR-expressing tumor cells in vivo using the therapeutic antibody cetuximab and noninvasive monitoring of treatment efficacy.

Several conjugation chemistries, including 1-ethyl3-(3-dimethylaminopropyl)carbodiimide hydrochloride or succinimidyl-4-( $N$-maleimidomethyl) cyclohexane-1carboxylate linker, have been used extensively in the conjugation of biomolecules to nanoparticles. ${ }^{29}$ The limitations of these conjugation methods are that the amino acid residues (such as lysine) in an antibody are randomly conjugated to these linkers, and the antigen-binding domains are not oriented towards antigen-presenting cells. These characteristics can reduce binding potency and thus affect therapeutic and/or diagnostic applications. In this work, we used periodate oxidation chemistry to achieve Fc-directed conjugation of iron oxide nanoparticles. ELISA demonstrated binding of the cet-PEG-dexSPIONs, like cetuximab, to EGFRvIII-ECD-hFc 
protein in a concentration-dependent manner. Notably, the binding affinity to EGFRvIII-ECD-hFc was 0.166 and $0.443 \mathrm{nM}$ for cetuximab and PEG-dexSPION-immobilized cetuximab, respectively, indicating that nanoparticle coupling retained the antibody-binding potency. Further, prevention of interactions between the nanoparticles and serum protein and subsequent capture by the reticuloendothelial system are requisites for cell targeting in vivo. PEG has been approved by the US Food and Drug Administration, and is the most widely used polymer in pharmaceutical formulations, foods, and cosmetics. ${ }^{36,37}$ PEG grafting has been widely used to improve the biocompatibility, solubility, and stability of nanoparticles in biological medium. PEGylation has been also reported to increase the circulation time of therapeutic drugs in blood and to avoid rapid recognition and clearance of nanoparticles by the reticuloendothelial system. ${ }^{38,39}$ Moreover, the use of a PEG spacer with a length longer than $250 \AA$ (with a corresponding molecular weight of 3,350 Da) is reported to allow effective cellular uptake of liposomes via folate receptor-mediated endocytosis, suggesting that a long intervening spacer is necessary for cellular recognition of the PEG-conjugated nanoparticles by overcoming the steric hindrance encountered at the cell surface..$^{40}$ Our data, including the results of the cellular iron uptake assay and MRI phantom study, support the notion that PEGylation prevents nonspecific uptake of PEG-dexSPIONs, thus providing the basis for ligand-mediated cellular uptake.

Hadjipanayis et $\mathrm{al}^{41}$ described a potential multifunctional magnetic nanoparticle, denoted as the EGFRvIIIAb-iron oxide nanoparticle (IONP), for selective MRI contrast enhancement of tumor cells and targeted therapy of infiltrative glioblastoma cells after convection-enhanced delivery. Elevated levels of cleaved caspase- 3 indicated that apoptosis might have contributed to the death of human glioblastoma cells and glioblastoma stem cell-containing neurospheres following treatment with EGFRvIIIAb-IONPs. However, transmission electron micrographs revealed significant nonspecific cellular surface binding and enhanced cellular uptake of the IONPs via endocytosis, which may have led to pronounced cytotoxicity in human glioblastoma cells. Moreover, the decreased level of phosphorylated AKT and lack of phosphorylated ERK in human glioblastoma cells treated with either free IONPs or EGFRvIIIAb-IONPs indicated that the therapeutic effect of the resulting EGFRvIIIAb-IONPs was contributed by the cytotoxicity of the IONPs per se rather than the anti-EGFRvIII antibody. In contrast, through Fc-directed conjugation of the therapeutic monoclonal antibody, cet-PEG-dexSPIONs, but not PEG-dexSPIONs, enhance the therapeutic effects of cetuximab. Cetuximab has been known to interact with the ectodomain of the EGFR to block ligand binding and to induce internalization and subsequent degradation of EGFR. ${ }^{42,43}$ Western blot analysis showed that cet-PEGdexSPIONs not only completely inhibited epidermal growth factor-induced EGFR phosphorylation but also significantly affected EGFR degradation when A431 cells were treated with cet-PEG-dexSPIONs compared with cetuximab alone. This finding demonstrates the rapid internalization and degradation of EGFR, which is consistent with the result reported by Bhattacharyya et al who demonstrated that conjugation of cetuximab to gold nanoparticles enhanced EGFR endocytosis in pancreatic cancer cell lines with variable EGFR expression. ${ }^{34}$ Their results using confocal microscopy and transmission electron microscopy confirmed rapid internalization and predominantly endosomal localization of these antibody-nanoparticle conjugates in EGFR-expressing cells via receptor-mediated endocytosis. The ability of cetuximabSPION conjugates to downregulate surface EGFR is clinically significant because attenuation of the membrane presentation of the receptor implies reduced signaling through its corresponding ligand. Our results for cellular iron uptake also support the notion of rapid endocytosis of cet-PEGdexSPIONs due to the significant increase in iron content of A431 cells within 30 minutes after treatment with $25 \mu \mathrm{g} / \mathrm{mL}$ of cet-PEG-dexSPIONs. The confocal images demonstrated accelerated cetuximab-induced EGFR endocytosis in cetPEG-dexSPION-treated A431 cells (data not shown). These altered EGFR endocytic dynamics may enhance the therapeutic antitumor efficacy of cet-PEG-dexSPIONs.

In addition, the conjugation of numerous antibody molecules to a single bead particle greatly enhances the avidity of the particle to target cells, thereby promoting the cross-linking of EGFR. It was recently reported that administration of two antibodies that specifically target to nonoverlapping epitopes on domain III of EGFR induced receptor clustering ${ }^{44}$ and reduced surface EGFR levels by up to $80 \%$ in many EGFRexpressing tumor cells. ${ }^{45}$ Enhanced EGFR internalization and degradation, mostly by preventing endosomal recycling of EGFR back to the cell surface, caused a marked reduction in downstream signaling and inhibited cell proliferation and migration. This result suggests that cross-linking of the EGFR may be an effective antitumor strategy. Further, the observed antitumor activity may be attributed to blocking of the nonkinase functions of EGFR, including protection against p53-upregulated modulator of apoptosis (PUMA)mediated apoptosis ${ }^{46,47}$ and prevention of autophagy through maintenance of glucose uptake. ${ }^{48}$ In accordance with these 
findings, the enhanced therapeutic effects of multivalent targeted nanoparticles caused by the enhanced endocytic rate of cet-PEG-dexSPIONs were supported by the more rapid downregulation of surface EGFR within 5 minutes after treatment in cet-PEG-dexSPION-treated A431 cells compared with cetuximab alone. We also observed significantly enhanced apoptosis in 32D/EGFR cells that were treated with $10 \mu \mathrm{g} \mathrm{Fe} / \mathrm{mL}$ cet-PEG-dexSPIONs (ie, $0.5 \mu \mathrm{g} / \mathrm{mL}$ cetuximab) in comparison with those treated with $2 \mu \mathrm{g} / \mathrm{mL}$ cetuximab alone (Figure S6B). Since iron oxide nanoparticles at low iron concentrations such as $10 \mu \mathrm{g} \mathrm{Fe} / \mathrm{mL}$ do not cause significant cell damage or death in macrophages by inducing cell cycle arrest and generation of reactive oxygen species, ${ }^{49,50}$ the marked increase in the portion of cells in the early apoptotic phase after 24 hours of treatment may be primarily attributed to the EGFR cross-linking effect of cet-PEG-dexSPIONs. However, when the SPION dose was increased (eg, to $50 \mu \mathrm{g} \mathrm{Fe} / \mathrm{mL}$ ), the high amount of iron accumulation inside the cells may also synergize the antitumor effect of cet-PEGdexSPIONs. To evaluate this possibility, the toxic effect of iron should be further evaluated by functionalizing the particles with cell-penetrating peptides.

$\mathrm{ADCC}$ is mediated by engagement of the Fc region of the antibody to the FcyRIIIA receptor on immune effector cells, such as natural killer cells or macrophages/monocytes, upon binding of tumor or viral antigen. Simultaneous binding of the antibody to target and immune effector cells is required, and results in cross-linking of FcyRIIIA followed by activation of FcyRIIIA signaling and release of proteins that mediate the killing of target cells such as perforin and of proteases such as granzymes. Our results demonstrated a reduction in the effect of cet-PEG-dexSPIONs on ADCC compared with an equivalent amount of cetuximab with respect to the lysis of A431 cells. It is possible that rapid internalization of cetPEG-dexSPIONs following binding to cells may reduce the availability of the antibodies for bridging target and immune effector cells. In addition, the contribution of glycosylation of monoclonal antibodies in ADCC has been reported. ${ }^{51}$ It is likely that the carbohydrate moieties of the antibody molecule were destroyed by periodate oxidation, resulting in decreasing ADCC activity of cet-PEG-dexSPIONs.

The improved therapeutic effect of cet-PEG-dexSPIONs is generally attributed to the EGFR cross-linking ability compared with cetuximab alone by enhancing the internalization of EGFR. This effect is influenced by the coupling ratio of cetuximab and SPIONs, ie, the number of antibody molecules on a nanoparticle. In this study, we used cetuximab in excess to maximize the conjugation efficiency. After antibody conjugation, only about $45 \%$ of the cetuximab molecules were grafted onto the particle surfaces (see Figure 2A). The amount of cetuximab on a SPION may be reduced and the coupling ratio between SPIONs and cetuximab be further optimized. Moreover, the ability of cet-PEG-dexSPIONs to trigger a decrease in EGFR expression levels upon various coupling ratios of SPIONs and cetuximab may be an interesting topic for evaluation.

\section{Conclusion}

We have demonstrated successful functionalization of dextran-coated SPIONs with the anti-EGFR therapeutic monoclonal antibody cetuximab via the dihydrazidePEG linker. The binding affinity of cet-PEG-dexSPIONs to EGFRvIII-ECD-hFc determined by ELISA was only slightly decreased compared with that of cetuximab, suggesting that the antigen binding sites remained free for targeting through the directional conjugation strategy. We discovered that cet-PEG-dexSPIONs not only inhibited epidermal growth factor-induced EGFR phosphorylation but also enhanced EGFR internalization and degradation. Moreover, there was a significant increase in apoptosis in EGFR-expressing tumor cells treated with cet-PEG-dexSPIONs. These therapeutic effects may be attributed to the multivalency of the resulting magnetic nanoprobes. Despite rapid internalization, the cet-PEG-dexSPIONs still had an ADCC effect with approximately $10.4 \%$ target cell lysis. In addition, the in vitro assay revealed active tumor targeting and rapid internalization via receptor-mediated endocytosis of the cet-PEG-dexSPIONs, enabling ultrasensitive tumor imaging. These results suggest that cet-PEG-dexSPIONs could be a potential MRI contrast agent for the diagnosis and treatment of cancer.

\section{Acknowledgments}

The authors thank the Taiwan Mouse Clinic (NSC 1022325-B-001-042) which is funded by the National Research Program for Biopharmaceuticals at the National Science Council of Taiwan for technical support with the molecular imaging experiment.

\section{Disclosure}

The authors report no conflicts of interest in this work.

\section{References}

1. Grandis JR, Sok JC. Signaling through the epidermal growth factor receptor during the development of malignancy. Pharmacol Ther. 2004; 102(1):37-46.

2. Harris AL, Nicholson S, Sainsbury R, Wright C, Farndon J. Epidermal growth factor receptor and other oncogenes as prognostic markers. J Natl Cancer Inst Monogr. 1992;(11):181-187. 
3. Ang KK, Berkey BA, Tu X, et al. Impact of epidermal growth factor receptor expression on survival and pattern of relapse in patients with advanced head and neck carcinoma. Cancer Res. 2002;62(24): 7350-7356.

4. Rubin Grandis J, Melhem MF, Gooding WE, et al. Levels of TGFalpha and EGFR protein in head and neck squamous cell carcinoma and patient survival. J Natl Cancer Inst. 1998;90(11):824-832.

5. Kim ES, Khuri FR, Herbst RS. Epidermal growth factor receptor biology (IMC-C225). Curr Opin Oncol. 2001;13(6):506-513.

6. Van Cutsem E, Kohne CH, Hitre E, et al. Cetuximab and chemotherapy as initial treatment for metastatic colorectal cancer. N Engl J Med. 2009; 360(14):1408-1417.

7. Van Cutsem E, Kohne CH, Lang I, et al. Cetuximab plus irinotecan, fluorouracil, and leucovorin as first-line treatment for metastatic colorectal cancer: updated analysis of overall survival according to tumor KRAS and BRAF mutation status. J Clin Oncol. 2011;29(15):2011-2019.

8. [No authors listed]. Cetuximab approved by FDA for treatment of head and neck squamous cell cancer. Cancer Biol Ther. 2006;5(4):340-342.

9. Mendelsohn J. Blockade of receptors for growth factors: an anticancer therapy - the fourth annual Joseph H Burchenal American Association for Cancer Research Clinical Research Award Lecture. Clin Cancer Res. 2000;6(3):747-753.

10. Ciardiello F, Tortora G. EGFR antagonists in cancer treatment. $N$ Engl J Med. 2008;358(11):1160-1174.

11. Fan Z, Masui H, Altas I, Mendelsohn J. Blockade of epidermal growth factor receptor function by bivalent and monovalent fragments of 225 anti-epidermal growth factor receptor monoclonal antibodies. Cancer Res. 1993;53(18):4322-4328.

12. Fan Z, Lu Y, Wu X, Mendelsohn J. Antibody-induced epidermal growth factor receptor dimerization mediates inhibition of autocrine proliferation of A431 squamous carcinoma cells. J Biol Chem. 1994; 269(44):27595-27602.

13. Prewett M, Rockwell P, Rockwell RF, et al. The biologic effects of C225, a chimeric monoclonal antibody to the EGFR, on human prostate carcinoma. J Immunother Emphasis Tumor Immunol. 1996;19(6):419-427.

14. Huang S-M, Bock JM, Harari PM. Epidermal growth factor receptor blockade with C225 modulates proliferation, apoptosis, and radiosensitivity in squamous cell carcinomas of the head and neck. Cancer Res. 1999;59(8):1935-1940.

15. Huang S-M, Harari PM. Modulation of radiation response after epidermal growth factor receptor blockade in squamous cell carcinomas: inhibition of damage repair, cell cycle kinetics, and tumor angiogenesis. Clin Cancer Res. 2000;6(6):2166-2174.

16. Bruns CJ, Harbison MT, Davis DW, et al. Epidermal growth factor receptor blockade with $\mathrm{C} 225$ plus gemcitabine results in regression of human pancreatic carcinoma growing orthotopically in nude mice by antiangiogenic mechanisms. Clin Cancer Res. 2000;6(5):1936-1948.

17. Perrotte $\mathrm{P}$, Matsumoto $\mathrm{T}$, Inoue $\mathrm{K}$, et al. Anti-epidermal growth factor receptor antibody $\mathrm{C} 225$ inhibits angiogenesis in human transitional cell carcinoma growing orthotopically in nude mice. Clin Cancer Res. 1999;5(2):257-265.

18. Wu X, Fan Z, Masui H, Rosen N, Mendelsohn J. Apoptosis induced by an anti-epidermal growth factor receptor monoclonal antibody in a human colorectal carcinoma cell line and its delay by insulin. J Clin Invest. 1995;95(4):1897-1905.

19. Peng D, Fan Z, Lu Y, DeBlasio T, Scher H, Mendelsohn J. Antiepidermal growth factor receptor monoclonal antibody 225 up-regulates p27KIP1 and induces G1 arrest in prostatic cancer cell line DU145. Cancer Res. 1996;56(16):3666-3669.

20. Bonner JA, Raisch KP, Trummell HQ, et al. Enhanced apoptosis with combination $\mathrm{C} 225$ /radiation treatment serves as the impetus for clinical investigation in head and neck cancers. J Clin Oncol. 2000;18 (21 Suppl 1):S47-S53.

21. Bandyopadhyay D, Mandal M, Adam L, Mendelsohn J, Kumar R. Physical interaction between epidermal growth factor receptor and DNA-dependent protein kinase in mammalian cells. J Biol Chem. 1998;273(3):1568-1573.
22. Naramura M, Gillies SD, Mendelsohn J, Reisfeld RA, Mueller BM. Therapeutic potential of chimeric and murine anti-(epidermal growth factor receptor) antibodies in a metastasis model for human melanoma. Cancer Immunol Immunother. 1993;37(5):343-349.

23. Kurai J, Chikumi H, Hashimoto K, et al. Antibody-dependent cellular cytotoxicity mediated by cetuximab against lung cancer cell lines. Clin Cancer Res. 2007;13(5):1552-1561.

24. Conde J, Bao C, Cui D, Baptista PV, Tian F. Antibody-drug gold nanoantennas with Raman spectroscopic fingerprints for in vivo tumour theranostics. J Control Release. 2014;183:87-93.

25. Karmani L, Labar D, Valembois V, et al. Antibody-functionalized nanoparticles for imaging cancer: influence of conjugation to gold nanoparticles on the biodistribution of $89 \mathrm{Zr}$-labeled cetuximab in mice. Contrast Media Mol Imaging. 2013;8(5):402-408.

26. Kao HW, Lin YY, Chen CC, et al. Biological characterization of cetuximab-conjugated gold nanoparticles in a tumor animal model. Nanotechnology [serial on the Internet]. Available from: http://iopscience. iop.org/0957-4484/25/29/295102/. Accessed February 28, 2015.

27. Ferrucci JT, Stark DD. Iron oxide-enhanced MR imaging of the liver and spleen: review of the first 5 years. AJR Am J Roentgenol. 1990; 155(5):943-950.

28. Mura S, Couvreur P. Nanotheranostics for personalized medicine. $A d v$ Drug Deliv Rev. 2012;64(13):1394-1416.

29. Yu MK, Park J, Jon S. Targeting strategies for multifunctional nanoparticles in cancer imaging and therapy. Theranostics. 2012;2(1):3-44.

30. Villanueva A, Canete M, Roca AG, et al. The influence of surface functionalization on the enhanced internalization of magnetic nanoparticles in cancer cells. Nanotechnology [serial on the Internet]. Available from: http://iopscience.iop.org/0957-4484/20/11/115103/. Accessed February 3, 2015.

31. LaConte LE, Nitin N, Zurkiya O, et al. Coating thickness of magnetic iron oxide nanoparticles affects $\mathrm{R} 2$ relaxivity. J Magn Reson Imaging. 2007;26(6):1634-1641.

32. Laurent S, Forge D, Port M, et al. Magnetic iron oxide nanoparticles: synthesis, stabilization, vectorization, physicochemical characterizations, and biological applications. Chem Rev. 2008;108(6):2064-2110.

33. Patel D, Lahiji A, Patel S, et al. Monoclonal antibody cetuximab binds to and down-regulates constitutively activated epidermal growth factor receptor vIII on the cell surface. Anticancer Res. 2007;27(5A): 3355-3366.

34. Bhattacharyya S, Bhattacharya R, Curley S, McNiven MA, Mukherjee P. Nanoconjugation modulates the trafficking and mechanism of antibody induced receptor endocytosis. Proc Natl Acad Sci US A. 2010;107(33): 14541-14546

35. Campbell KS, Inventor; Fox Chase Cancer Center, assignee. Genetically modified human natural killer cell lines. United States patent US 8313943. November 20, 2012.

36. Bailon P, Won CY. PEG-modified biopharmaceuticals. Expert Opin Drug Deliv. 2009;6(1):1-16.

37. Fuertges F, Abuchowski A. The clinical efficacy of poly(ethylene glycol)-modified proteins. J Control Release. 1990;11(1-3):139-148.

38. Zalipsky S. Chemistry of polyethylene glycol conjugates with biologically active molecules. Adv Drug Deliv Rev. 1995;16(2-3):157-182.

39. Papahadjopoulos D, Allen TM, Gabizon A, et al. Sterically stabilized liposomes: improvements in pharmacokinetics and antitumor therapeutic efficacy. Proc Natl Acad Sci U S A. 1991;88(24):11460-11464.

40. Lee RJ, Low PS. Delivery of liposomes into cultured KB cells via folate receptor-mediated endocytosis. J Biol Chem. 1994;269(5): 3198-3204.

41. Hadjipanayis CG, Machaidze R, Kaluzova M, et al. EGFRvIII antibody-conjugated iron oxide nanoparticles for magnetic resonance imaging-guided convection-enhanced delivery and targeted therapy of glioblastoma. Cancer Res. 2010;70(15):6303-6312.

42. Sunada H, Magun BE, Mendelsohn J, MacLeod CL. Monoclonal antibody against epidermal growth factor receptor is internalized without stimulating receptor phosphorylation. Proc Natl Acad Sci US A. 1986; 83(11):3825-3829. 
43. Jaramillo ML, Leon Z, Grothe S, Paul-Roc B, Abulrob A, O'Connor McCourt M. Effect of the anti-receptor ligand-blocking 225 monoclonal antibody on EGF receptor endocytosis and sorting. Exp Cell Res. 2006; 312(15):2778-2790.

44. Friedman LM, Rinon A, Schechter B, et al. Synergistic down-regulation of receptor tyrosine kinases by combinations of mAbs: implications for cancer immunotherapy. Proc Natl Acad Sci U S A. 2005;102(6): 1915-1920.

45. Spangler JB, Neil JR, Abramovitch S, et al. Combination antibody treatment down-regulates epidermal growth factor receptor by inhibiting endosomal recycling. Proc Natl Acad Sci U S A. 2010;107(30): 13252-13257.

46. Sun Q, Ming L, Thomas SM, et al. PUMA mediates EGFR tyrosine kinase inhibitor-induced apoptosis in head and neck cancer cells. Oncogene. 2009;28(24):2348-2357.

47. Zhu H, Cao X, Ali-Osman F, Keir S, Lo HW. EGFR and EGFRvIII interact with PUMA to inhibit mitochondrial translocalization of PUMA and PUMA-mediated apoptosis independent of EGFR kinase activity. Cancer Lett. 2010;294(1):101-110.
48. Weihua Z, Tsan R, Huang WC, et al. Survival of cancer cells is maintained by EGFR independent of its kinase activity. Cancer Cell. 2008;13(5):385-393.

49. Park EJ, Choi DH, Kim Y, et al. Magnetic iron oxide nanoparticles induce autophagy preceding apoptosis through mitochondrial damage and ER stress in RAW264.7 cells. Toxicol In Vitro. 2014;28(8):1402-1412.

50. Naqvi S, Samim M, Abdin M, et al. Concentration-dependent toxicity of iron oxide nanoparticles mediated by increased oxidative stress. Int J Nanomedicine. 2010;5:983-989.

51. Arnold JN, Wormald MR, Sim RB, Rudd PM, Dwek RA. The impact of glycosylation on the biological function and structure of human immunoglobulins. Annu Rev Immunol. 2007;25:21-50. 


\section{Supplementary materials}

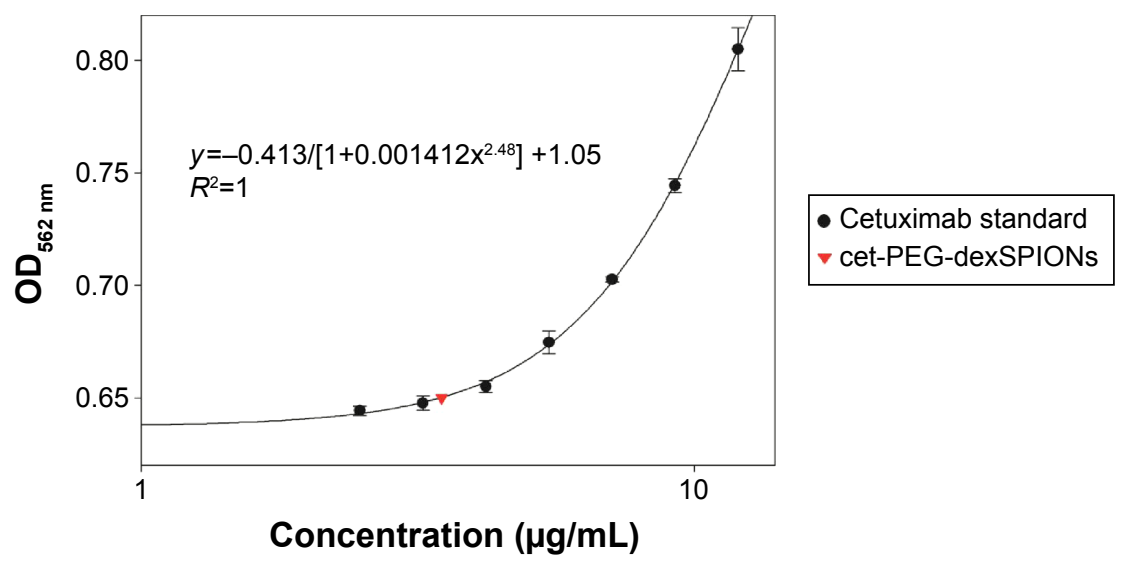

Figure SI Quantitation of immobilized cetuximab using the micro bicinchoninic acid protein assay. Cetuximab solution supplemented with an amount of iron equal to that in the synthesized cet-PEG-dexSPION sample, ranging from 0 to $12 \mu \mathrm{g} / \mathrm{mL}$, was used as a standard curve. The values for the OD at $562 \mathrm{~nm}$ were plotted against the concentration of cetuximab. The results are presented as the mean \pm standard deviation of three determinations.

Abbreviations: cet, cetuximab; dex, dextran; PEG, polyethylene glycol; SPIONs, superparamagnetic iron oxide nanoparticles; OD, optical density.

dexSPIONs

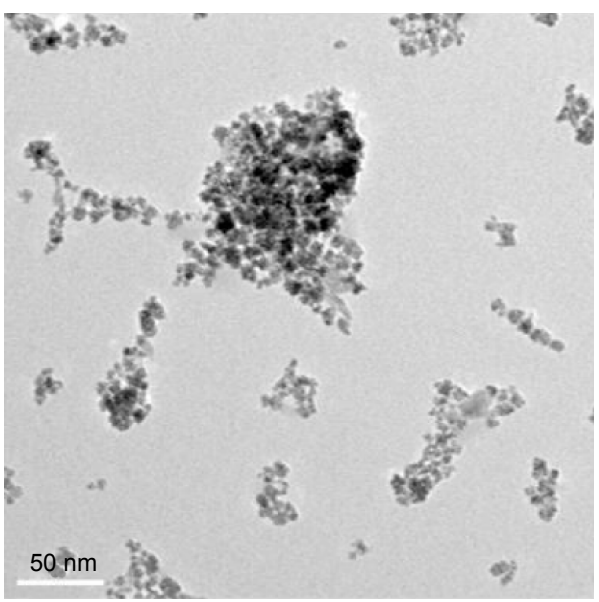

PEG-dexSPIONs

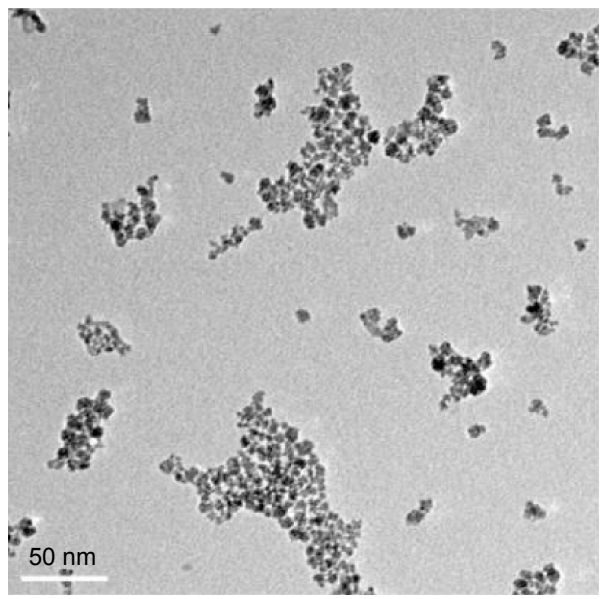

ses

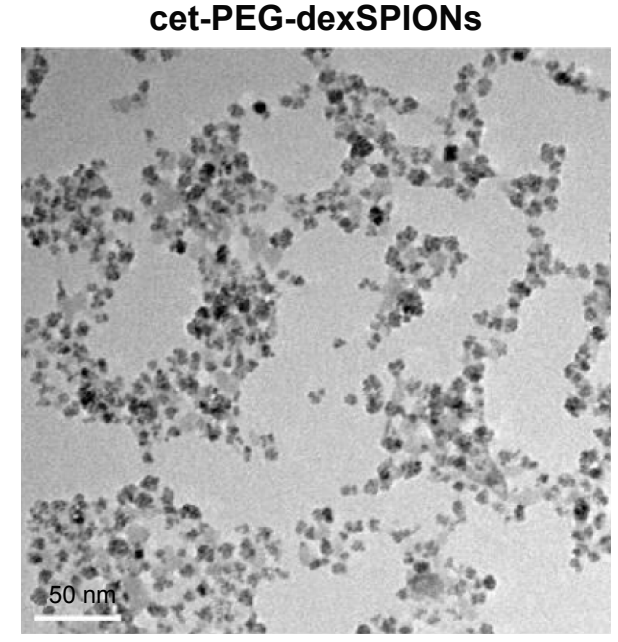

Figure S2 Transmission electron micrographs of dexSPIONs, PEG-dexSPIONs, and cet-PEG-dexSPIONs. The average size of particles estimated from the transmission electron images was about $10-20 \mathrm{~nm}$. Scale bar, $50 \mathrm{~nm}$.

Abbreviations: cet, cetuximab; dex, dextran; PEG, polyethylene glycol; SPIONs, superparamagnetic iron oxide nanoparticles. 
A
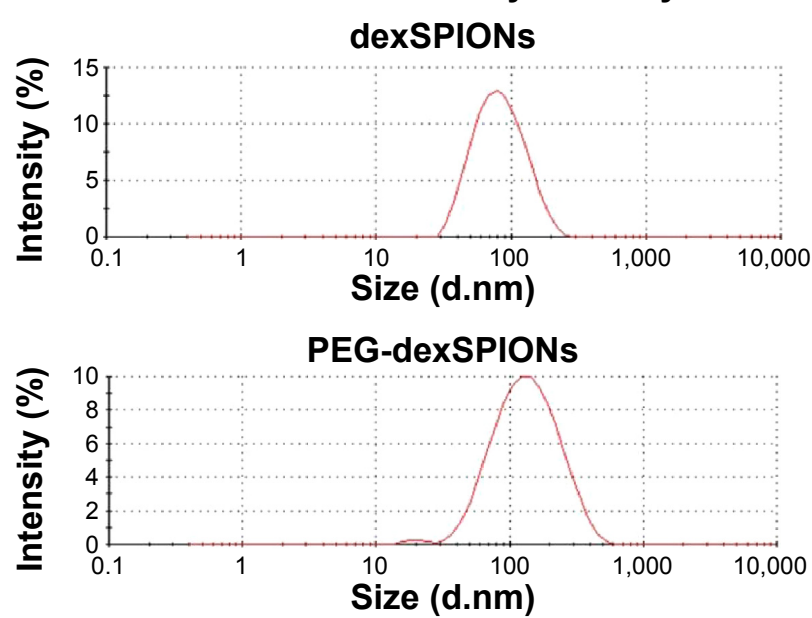

cet-PEG-dexSPIONs

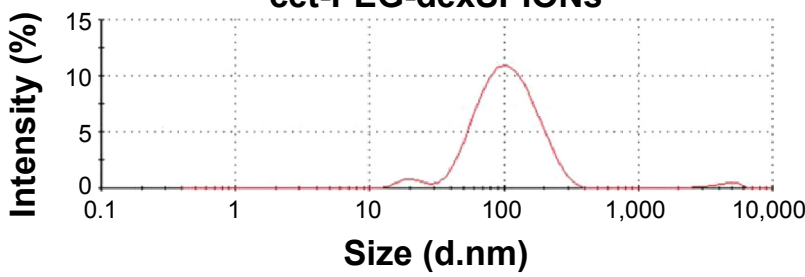

B

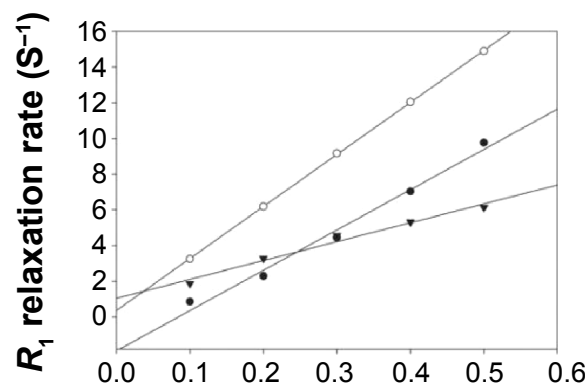

Fe concentration (mM)

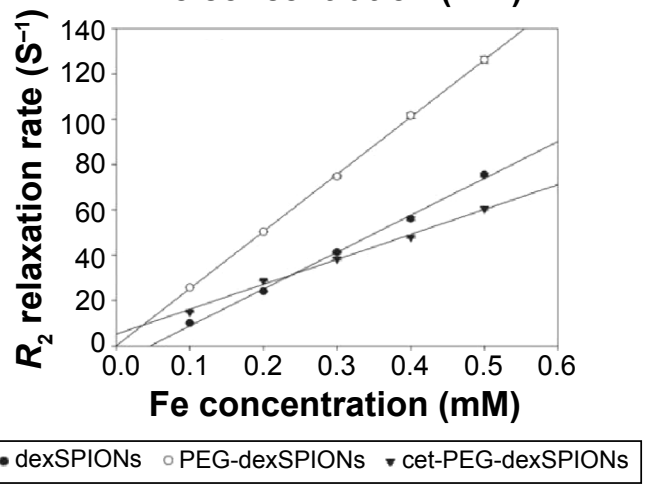

Figure S3 Characterization of the synthesized SPIONs.

Notes: (A) Hydrodynamic diameter of iron oxide nanoparticles determined by dynamic light scattering analysis. (B) Relaxivity values for the dexSPIONs and the synthesized nanoprobes. The longitudinal relaxation time $\left(T_{1}\right)$ and transverse relaxation time $\left(T_{2}\right)$ were measured at $37^{\circ} \mathrm{C}$ using a Bruker Minispec mq20 nuclear magnetic resonance analyzer $(20 \mathrm{mHz})$ at iron concentrations of 0.1 to $0.5 \mathrm{mM}$ for dexSPIONs, PEG-dexSPIONs, and cet-PEG-dexSPIONs, as measured immediately after vortexing in water solution. The longitudinal $\left(r_{1}\right)$ and transverse $\left(r_{2}\right)$ relaxivities of the iron oxide particles were determined based on the slopes of the linear regression of the longitudinal relaxation rate $R_{1}$ $\left(\mathrm{I} / \mathrm{T}_{1}\right)$ or transverse relaxation rate $\mathrm{R}_{2}\left(\mathrm{I} / \mathrm{T}_{2}\right)$ versus the iron concentration. The estimated relaxivity values for dexSPIONs were $r_{1}=22.575 \mathrm{mM} \mathrm{M}^{-1} \cdot \mathrm{s}^{-1}$ (correlation coefficient of 0.9866 ), $r_{2}=162.8 \mathrm{mM}^{-1} \cdot \mathrm{s}^{-1}$ (correlation coefficient of 0.9972 ); for PEG-dexSPIONs, $r_{1}=29.18 \mathrm{mM}^{-1} \cdot \mathrm{s}^{-1}$ (correlation coefficient of 0.9999 ), $r_{2}=252.25 \mathrm{mM}{ }^{-1} \cdot \mathrm{s}^{-1}$ (correlation coefficient of 0.9998 ); for cet-PEG-dexSPIONs, $r_{1}=10.586 \mathrm{mM}^{-1} \cdot \mathrm{s}^{-1}$ (correlation coefficient of 0.9808$), r_{2}=110.08 \mathrm{mM}^{-1} \cdot \mathrm{s}^{-1}$ (correlation coefficient of $0.995 \mathrm{I}$ ). Abbreviations: cet, cetuximab; dex, dextran; PEG, polyethylene glycol; SPIONs, superparamagnetic iron oxide nanoparticles.

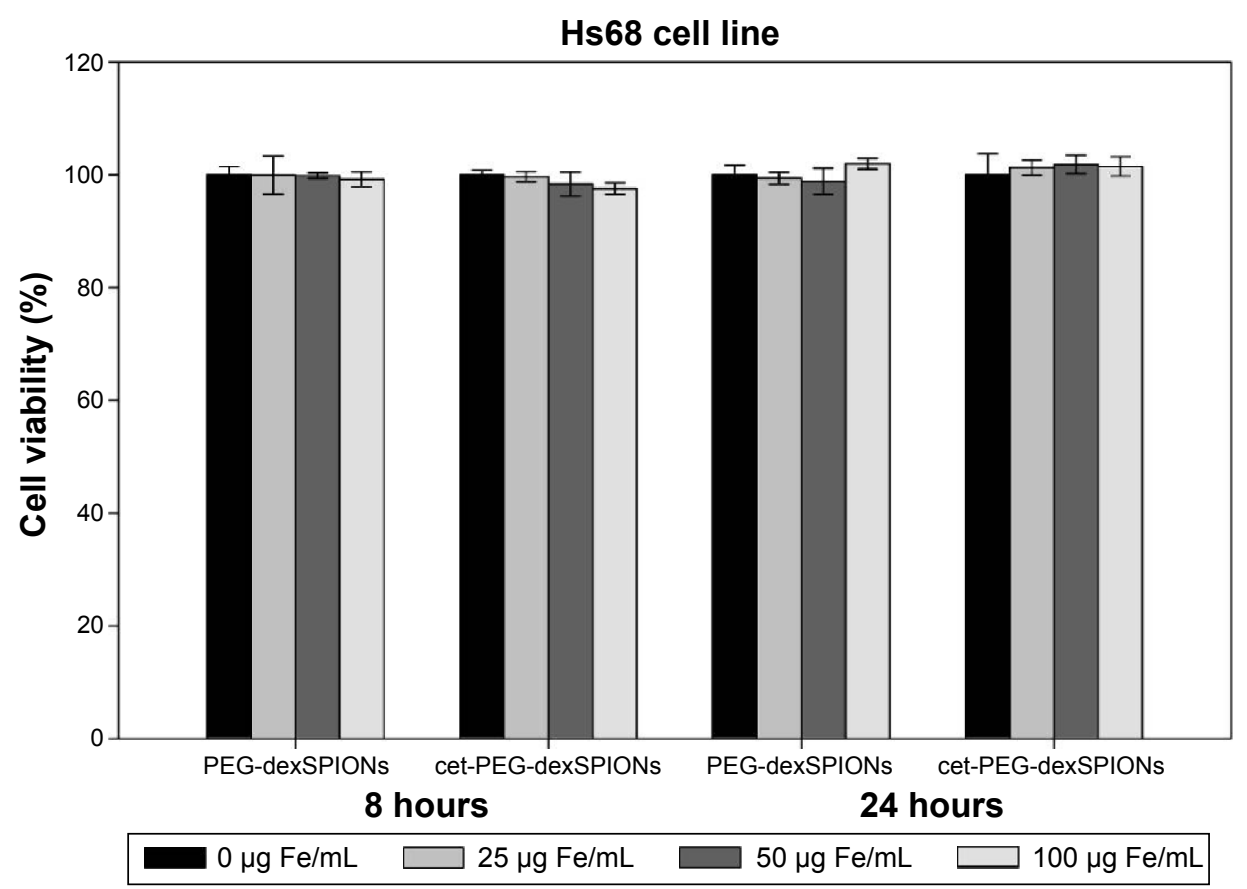

Figure S4 In vitro cytotoxicity of PEG-dexSPIONs and cet-PEG-dexSPIONs to the Hs68 cell line. The Hs68 cells were incubated with PEG-dexSPIONs or cet-PEGdexSPIONs at iron concentrations ranging from 25 to $100 \mu \mathrm{g} \mathrm{Fe} / \mathrm{mL}$ for 8 and 24 hours. Cytotoxicity was assessed by the Alamar Blue cytotoxicity assay. Results are presented as the mean \pm standard deviation of four replicates.

Abbreviations: cet, cetuximab; dex, dextran; PEG, polyethylene glycol; SPIONs, superparamagnetic iron oxide nanoparticles. 


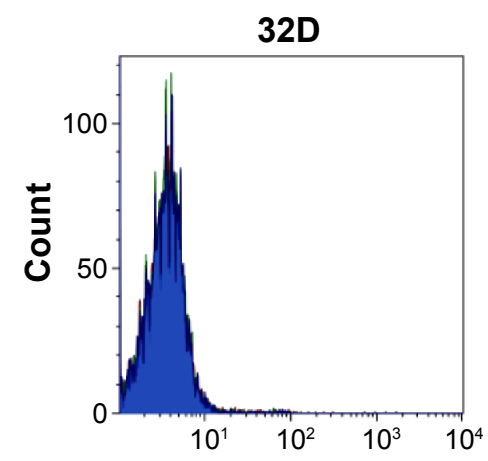

EGFR expression
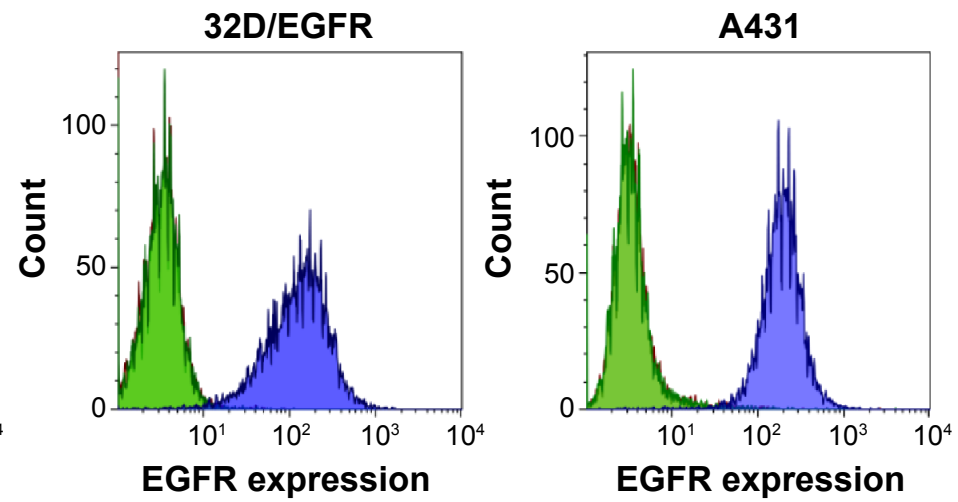

PE-conjugated mouse anti-human EGFR mAb $\square$ PE-conjugated mouse lgG2b $\kappa$ isotype control $\square$ Cell only

Figure S5 EGFR expression levels on the surface of tumor cell lines. EGFR expression on the surface of tumor cell lines was examined by flow cytometry using PE-conjugated mouse anti-human EGFR mAb. A PE-conjugated mouse lgG2b $\kappa$ isotype served as a negative control. The cell surface expression levels of EGFR were A43I > 32D/EGFR. In contrast, 32D cells were devoid of EGFR.

Abbreviations: cet, cetuximab; dex, dextran; EGFR, epidermal growth factor receptor; PEG, polyethylene glycol; SPIONs, superparamagnetic iron oxide nanoparticles; PE, phycoerythrin; mAb, monoclonal antibody; Ig, immunoglobulin.
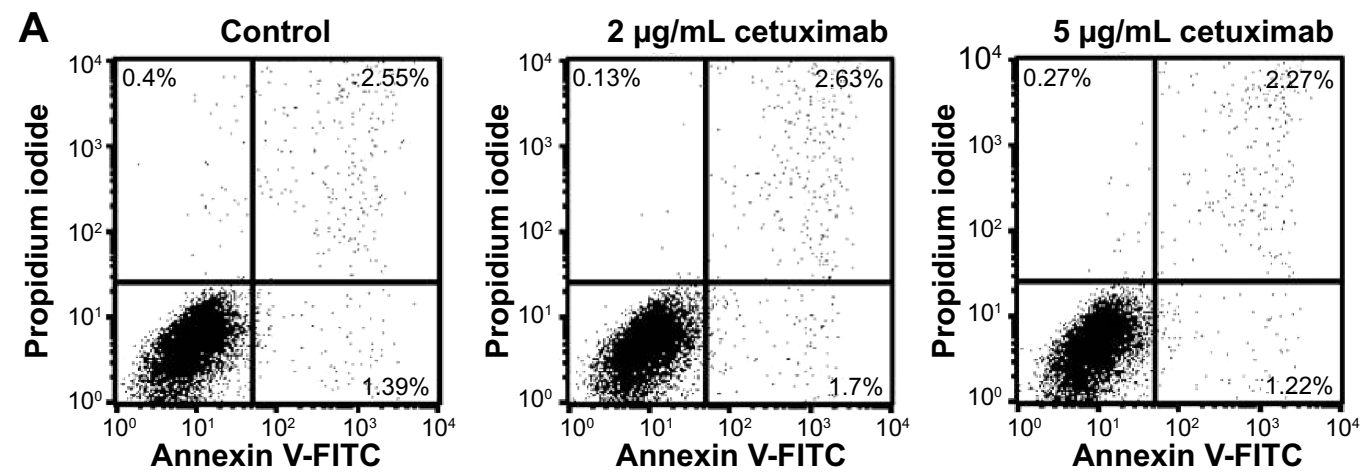

$10 \mu \mathrm{g} \mathrm{Fe} / \mathrm{mL}$ cet-PEG-dexSPIONs
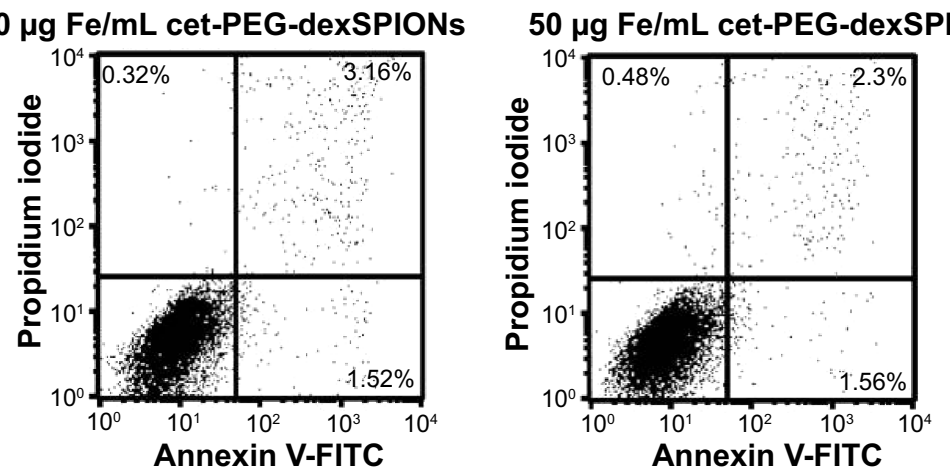

$10 \mu \mathrm{g} \mathrm{Fe} / \mathrm{mL}$ PEG-dexSPIONs
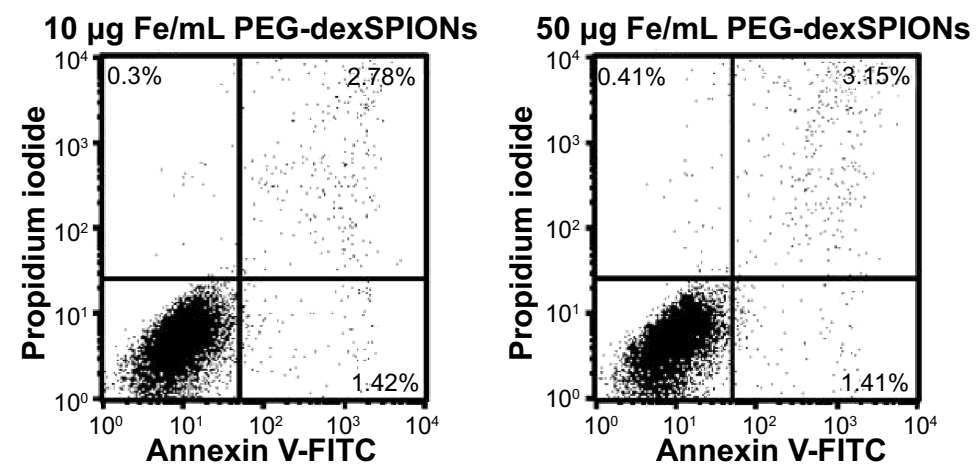

Figure S6 (Continued) 

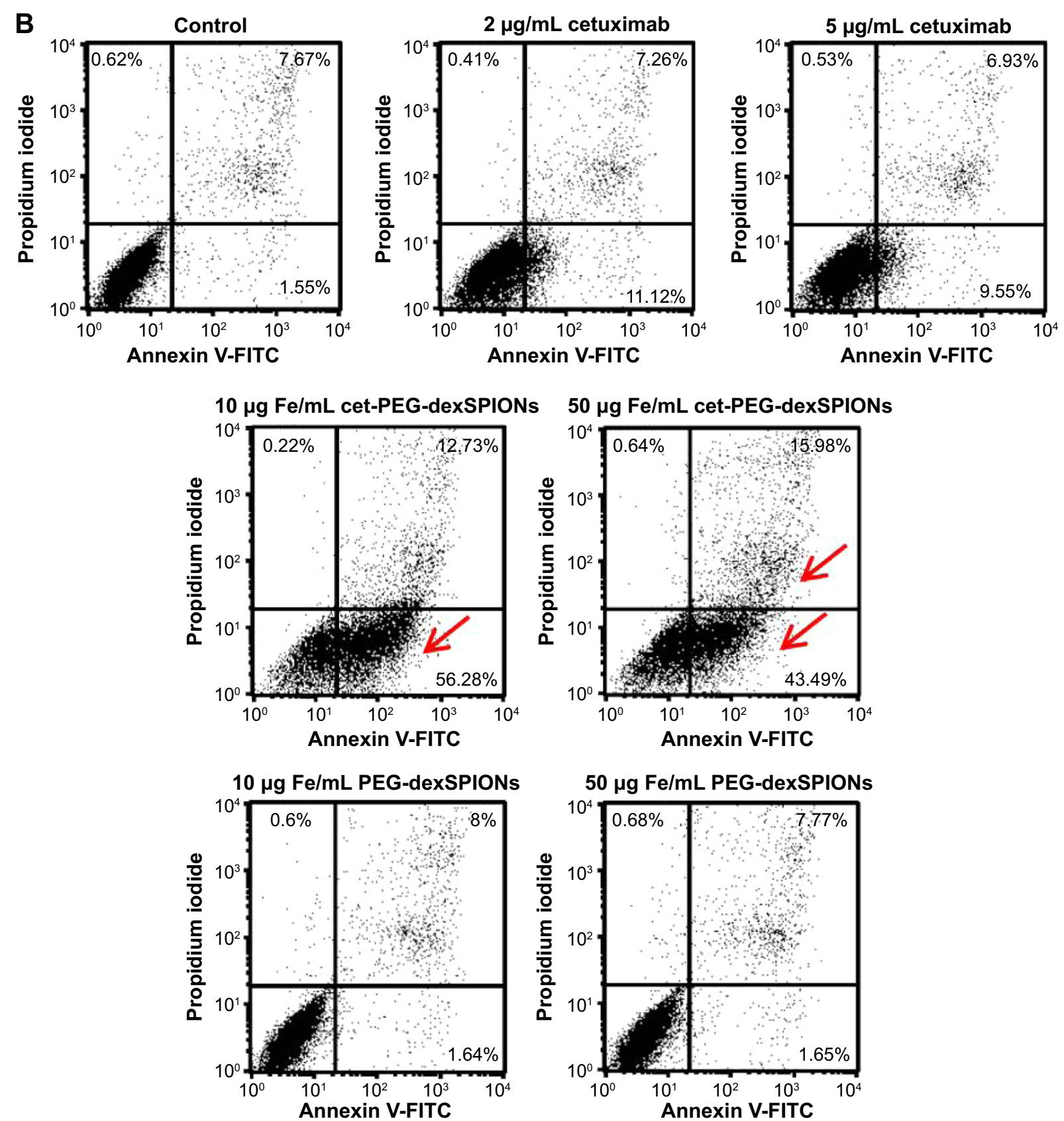

Figure S6 (Continued) 

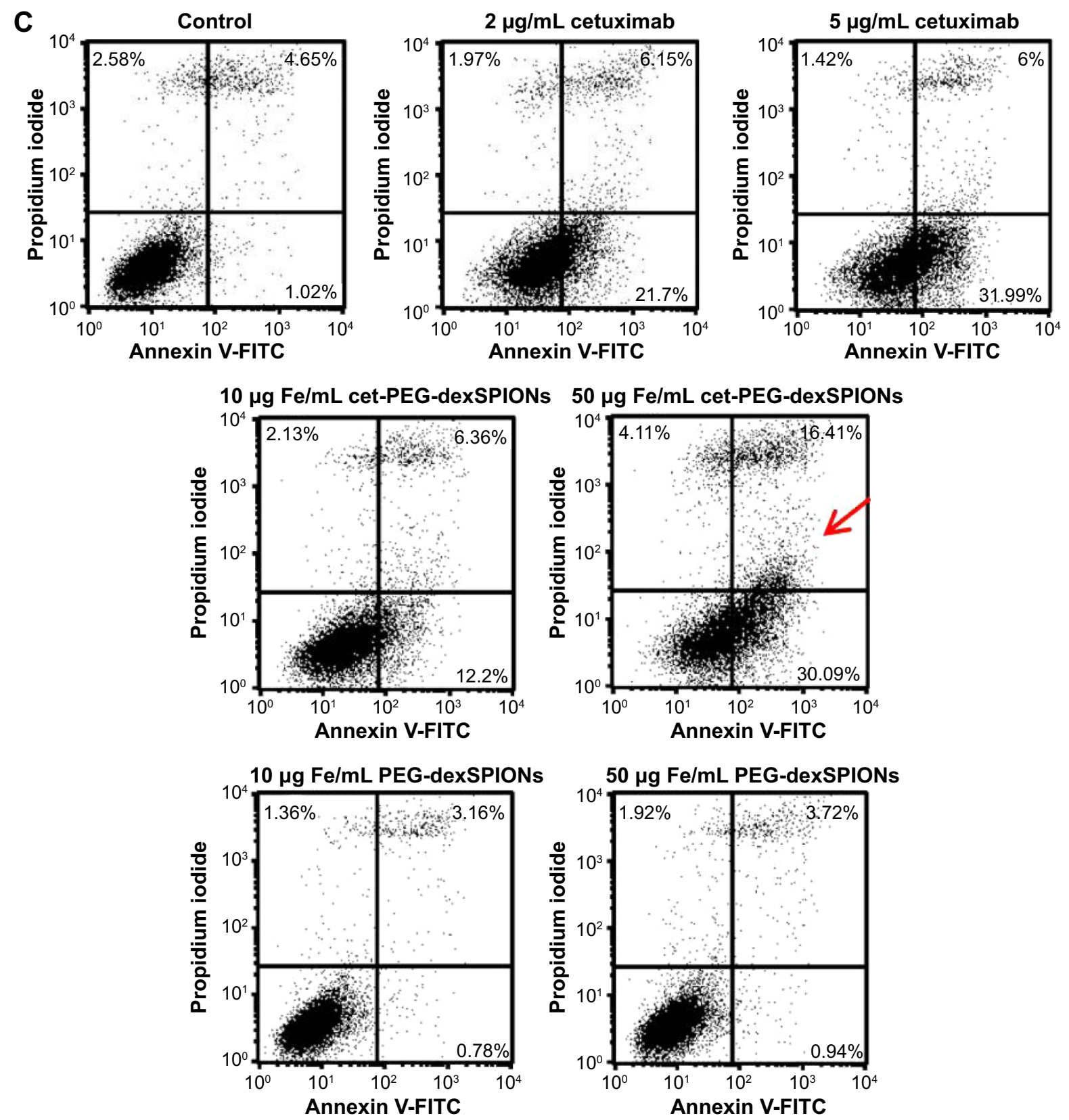

Figure S6 Apoptosis-inducing activity of cet-PEG-dexSPIONs in differential EGFR-expressing cell lines analyzed by an Annexin V-FITC/propidium iodide assay.

Notes: 32D (A), 32D/EGFR (B) and A43I (C) cells ( $1 \times 10^{5}$ cells) were incubated in $0.5 \mathrm{~mL}$ of complete medium containing cetuximab ( 2 or $\left.5 \mu \mathrm{g} / \mathrm{mL}\right)$ or iron oxide nanoparticles ( 25 or $50 \mu \mathrm{g} \mathrm{Fe} / \mathrm{mL}$ ). Tumor cell lines with different levels of EGFR expression ( $1 \times 10^{5}$ cells) were incubated in $0.5 \mathrm{~mL}$ of complete medium containing cetuximab ( 2 or $5 \mu \mathrm{g} / \mathrm{mL}$ ) or iron oxide nanoparticles ( 25 or $50 \mu \mathrm{g} \mathrm{Fe} / \mathrm{mL}$ ). After incubation for 24 hours, these cells were harvested and double-stained with FITC-conjugated Annexin-V and propidium iodide. The cell populations as a percent of the total cells were assessed by flow cytometry. The arrow indicates a significant increase in the cell population after the cells were treated with cet-PEG-dexSPIONs at the indicated concentrations. Annexin V-FITC-negative/propidium iodide-negative, viable cells; Annexin V-FITC-positive/propidium iodide-negative, early apoptotic cells; Annexin V-FITC-negative/propidium iodide-positive, dead cells; Annexin V-FITC-positive/propidium iodide-positive, late apoptotic cells and necrotic cells.

Abbreviations: cet, cetuximab; dex, dextran; EGFR, epidermal growth factor receptor; FITC, fluorescein isothiocyanate; PEG, polyethylene glycol; SPIONs, superparamagnetic iron oxide nanoparticles. 

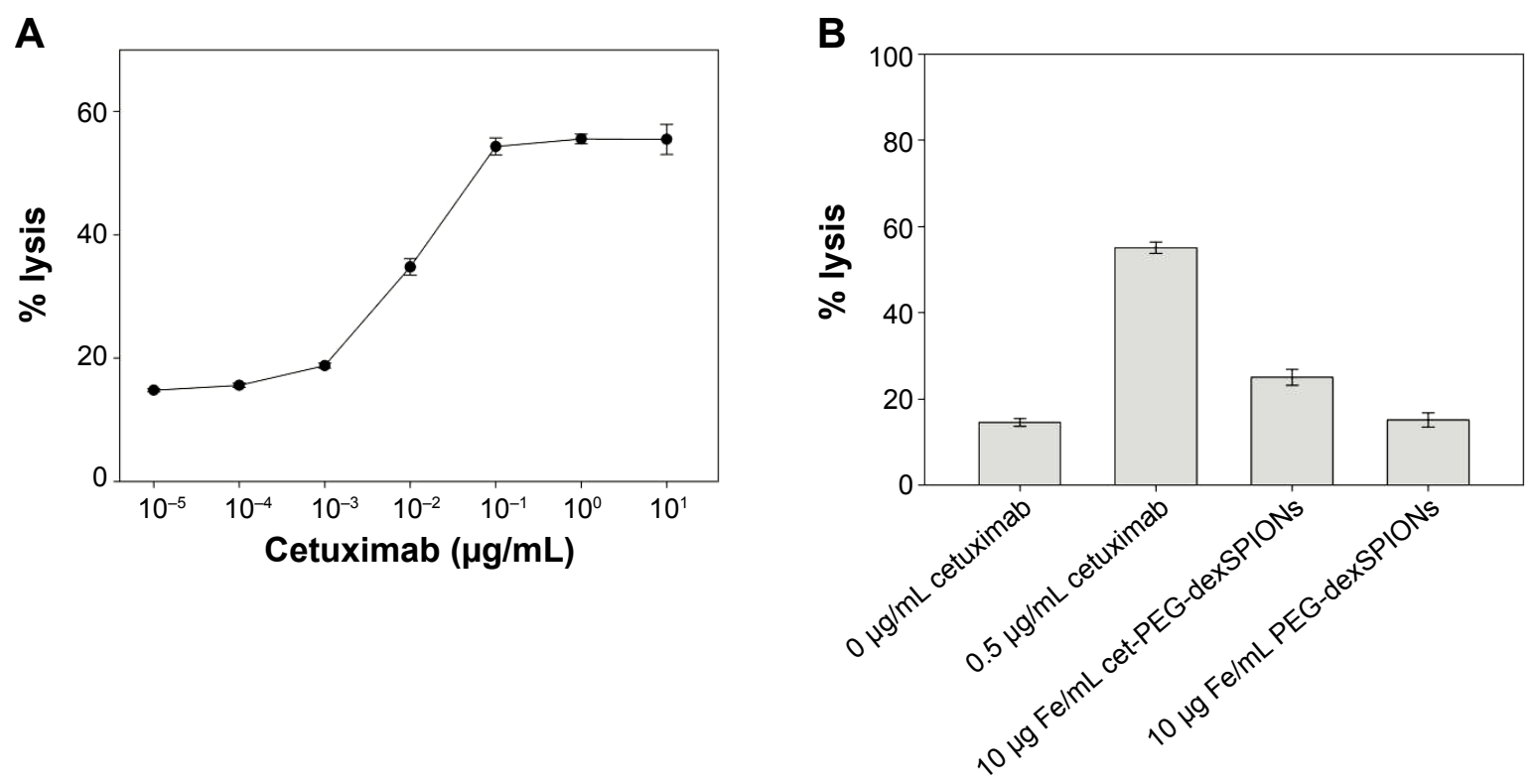

Figure S7 ADCC activity against A43I cells mediated by cetuximab.

Notes: (A) Concentration-dependent curve of cetuximab-mediated ADCC activity against A43I cells by NK-92-hCDI6V-GFP cells. A43I cells were incubated with NK92-hCD I6V-GFP cells at an E:T ratio of 10 to I along with indicated concentrations of cetuximab $(0.0000 \mathrm{I}-10 \mu \mathrm{g} / \mathrm{mL})$. The ADCC activity was determined based on the 4-hour lactate dehydrogenase release into the supernatant by A43I cells. The results are presented as the percent cell lysis in the antibody-treated groups compared with $100 \%$ lysis in the $1 \%$ Triton X-100 lysis buffer-treated group. (B) ADCC activity of cet-PEG-dexSPIONs against A43I cells by NK-92-hCDI6V-GFP cells. A43I cells were incubated in the absence or presence of $10 \mu \mathrm{g} \mathrm{Fe} / \mathrm{mL}$ synthesized iron oxide nanoparticles. Cetuximab $0.5 \mu \mathrm{g} / \mathrm{mL}$ served as a positive control. The data are representative of three independent experiments and are presented as the mean \pm standard deviation of four determinations.

Abbreviations: ADCC, antibody-dependent cell-mediated cytotoxicity; cet, cetuximab; dex, dextran; PEG, polyethylene glycol; SPIONs, superparamagnetic iron oxide nanoparticles.

\section{Publish your work in this journal}

The International Journal of Nanomedicine is an international, peerreviewed journal focusing on the application of nanotechnology in diagnostics, therapeutics, and drug delivery systems throughout the biomedical field. This journal is indexed on PubMed Central, MedLine, CAS, SciSearch ${ }^{\circledR}$, Current Contents ${ }^{\circledR} /$ Clinical Medicine,
Journal Citation Reports/Science Edition, EMBase, Scopus and the Elsevier Bibliographic databases. The manuscript management system is completely online and includes a very quick and fair peer-review system, which is all easy to use. Visit http://www.dovepress.com/ testimonials.php to read real quotes from published authors. 